\title{
Solar Cycle Variations of the First-Degree Spherical Harmonic Components of the Geomagnetic Field
}

\author{
Takesi YukutAKE ${ }^{\dagger *}$ and Joseph C. CAIN ${ }^{* *}$ \\ *CIRES, University of Colorado, Boulder, Colorado, U.S.A. \\ *Colorado School of Mines, Golden, Colorado, U.S.A. \\ **U.S. Geological Survey, Denver, Colorado, U.S.A.
}

(Received October 25, 1978; Revised January 27, 1979)

Spherical harmonic analyses were made of the annual mean values of the geomagnetic components at 34 observatories for the period from 1940 to 1973. Annual values of the Gauss coefficients were separated into parts of external and internal origin. The external part of the first-degree component is parallel to the internal dipole and changes with solar activity. The maximum change from peak to trough amounts to 47 nanoteslas during this period. During one solar cycle, two peaks appear in the variation of this component. The first peak corresponds to the period of the maximum solar activity, the second at the declining stage of the cycle.

Examination of the time variations of the first-degree components was extended to an analysis for 1900 to 1973 based on annual means from 21 observatories. Although its amplitude is small, the internal part changes with a three-year time lag behind the external field change. After Fourier analyses of the time series of both the internal and external components, their ratio was computed for the 10-year-period band. The modulus of the internal/external ratio $(i / e)$ was obtained to be 0.32 and the phase angle (internal-external) to be $105^{\circ}$.

\section{Introduction}

Variations in the geomagnetic field are strongly affected by solar phenomena. Various indices of the geomagnetic activity change with the solar cycle. However, the nature of the geomagnetic variation associated with the solar cycle is not yet fully understood. In this paper, year-to-year variation of the geomagnetic field caused by magnetic effects associated with solar activity is examined by expanding the annual mean values of the $X, Y$, and $Z$ components at selected observatories into spherical harmonic series.

The simplest way of expressing the solar cycle variation is to assume a

† On leave from the Earthquake Research Institute, University of Tokyo. Guest worker NOAA/EDS, National Geophysical and Solar-Terrestrial Data Center. 
sinusoid with a period of 11 years (Yukutake, 1965). However, this is too crude an approximation to represent the magnetic response to the solar activity. Courtillot and LeMouËL (1976) obtained a better representation of the geomagnetic changes by making use of the second and the third harmonics of the 11-year period (as well as the fundamental mode). Recently, HARWOoD and MALIN (1977) tried to correlate the geomagnetic variation with the change in relative sunspot number by making use of a linear combination of annual values of sunspot numbers and their annual rate of change. Although sunspot number is one of the best indices to measure solar activity, the phenomena taking place at the surface of the sun are not so simple as they can be described by a single index. From examination of magnetic indices, it has been recently inferred that two peaks exist in geomagnetic activity during one solar cycle (OL', 1971; Sutorik and Cruickshank, 1977; Meyers and Allen, 1977). These are longterm variations in the frequency of rather short-term magnetic disturbances, whereas the present paper attempts to investigate the long-term change in the field itself. In this paper, the first-degree components of external origin are first extracted without referring to the sunspot number.

Investigation of the response of the Earth to the external field variations provides important information regarding the distribution of electrical conductivity within the Earth. Since the penetration becomes deeper as the period of the phenomena becomes longer, analysis of the long-term variation of the external field is particularly important to obtain an estimate of the conductivity in the deeper part of the mantle. Many efforts have been made to separate the solar cycle variations of the geomagnetic field into external and internal parts (Yukutake, 1965; Courtillot and LeMouël, 1976; Alldredge, 1976; HARWOod and MALIN, 1977). Accurate extraction of the internal components is more difficult than that of the external parts, because of the small amplitude of the former. Accumulation of accurate data over a long period is required to make a reliable separation. Because of the limited number of observatories which can supply reliable data over several solar cycles, the separation of the external and the internal parts is not yet conclusive. Nevertheless the internal field variation related to the solar activity was extracted by a spherical harmonic analysis of annual mean values of the observatory data.

\section{The Process of the Analysis}

Two approaches can be used in extracting solar cycle variations from the annual mean values of the observatory data. One is to first make a spectrum analysis of the observed secular changes at individual observatories to extract solar cycle variations with specific periods, and then make a spherical har- 
monic analysis of these extracted variations to determine their global nature (Yukutake, 1965; Courtillot and LeMouël, 1976; Alldredge, 1976; HARWOOD and MALIN, 1977). This approach is a kind of frequency domain analysis. The other approach is to first employ a spherical harmonic analysis of the annual mean values and obtain the spherical harmonic coefficients for the successive years. The annual values of the Gauss coefficients thus obtained are then subjected to a spectral analysis (YukUtake, 1973; Alldredge et al., 1979).

If an anomalously large solar cycle variation exists at a specific observatory, the first method may detect the anomaly much more easily. However, the yearto-year variation of the global feature is hard to examine by this method, since detailed features of the variation have already been decomposed into a frequency domain at the first step. On the other hand, the second approach can reveal the year-to-year variation of the dipole field caused by the solar activity. The second approach is adopted in this paper.

\section{The Data}

For detecting small fluctuations in the Gauss coefficients it is desirable to have the data as uniformly distributed in space and as continuous in time as possible. As will be shown in later sections, alterations of the low-order terms by aliasing from neglected higher order coefficients can be large compared to the variations being sought. Thus, it is best to avoid changes in baselines, sites of the stations, or number of stations.

Since there are large areas of the earth not represented by any magnetic observatory, any spherical harmonic analysis of observatory data alone can contribute only to a global knowledge of the lowest order coefficients. By so limiting the analysis it is possible to obtain meaningful results from a small subset of the total data available. The data from 34 observatories covering the period 1940-1973 were selected as being sufficient to estimate the changes in the first few degrees and orders of harmonic terms. The geographic positions of these stations are listed in Table 1 and are illustrated in Fig. 1 by the circles. As can be seen here the largest gaps between stations are of the order of $90^{\circ}$, corresponding to $n=4$ in the harmonic series. Although there are many excellent observatories elsewhere, especially in Europe, their addition would not close these gaps and could tend to bias the results to the areas of greater observatory density. Such a biased result could be less representative of the global variation than that determined from fewer but more evenly distributed locations.

The discontinuities caused by movement of the site or change in standards were corrected using the constants provided by the individual observatories. 
Table 1. List of observatories.

\begin{tabular}{lllrrr}
\hline \multirow{2}{*}{ Code } & & & \multicolumn{3}{c}{ Geographic coordinates } \\
\cline { 3 - 5 } & & & Latitude & Longitude & Altitude $(\mathrm{km})$ \\
\hline 0 & GDH & Godhavn & 69.240 & -53.522 & .01 \\
0 & SOD & Sodankylä & 67.368 & 26.630 & .18 \\
0 & YAK & Yakutsk & 62.017 & 129.717 & .10 \\
1 & VOY & Voyeykovo & 59.950 & 30.705 & .07 \\
1 & SIT & Sitka & 57.058 & -135.325 & .02 \\
1 & VDA & Vysokaya-Dubrava & 56.733 & 61.067 & .29 \\
0 & MNK & Meanook & 54.617 & -113.333 & .68 \\
0 & RSV & Rude Skov & 55.843 & 12.457 & .05 \\
0 & ESK & Eskdalemuir & 55.317 & -3.200 & .24 \\
1 & PTY & Patrony & 52.167 & 104.450 & .54 \\
0 & FUR & Fürstenfeldbruck & 48.165 & 11.277 & .57 \\
1 & CLF & Chambon-la-Forêt & 48.023 & 2.260 & .15 \\
0 & YSS & Yuzhno Sakhalinsk & 46.950 & 142.717 & .07 \\
2 & AGN & Agincourt & 43.783 & -79.267 & .06 \\
1 & DUS & Dusheti & 42.092 & 44.705 & .98 \\
0 & TKT & Tashkent & 41.333 & 69.617 & .81 \\
0 & COI & Coimbra & 40.222 & -8.422 & .10 \\
0 & FRD & Fredericksburg & 38.205 & -77.373 & .07 \\
1 & SMG & San Miguel & 37.773 & -25.653 & .18 \\
1 & KAK & Kakioka & 36.230 & 140.190 & .03 \\
1 & TUC & Tucson & 32.247 & -110.833 & .77 \\
1 & MLT & Misallat & 29.515 & 30.892 & .12 \\
1 & HON & Honolulu & 21.320 & -158.002 & .00 \\
0 & TED & Teoloyucan & 19.747 & -99.182 & 2.28 \\
1 & ABG & Alibag & 18.638 & 72.872 & .01 \\
1 & SJG & San Juan & 18.117 & -66.150 & .10 \\
2 & KUY & Kuyper & -6.033 & 106.733 & .00 \\
2 & MRI & Mauritius & -20.093 & 57.553 & .05 \\
0 & HUA & Huancayo & -12.045 & -75.340 & 3.31 \\
1 & API & Apia & -13.807 & -171.775 & .00 \\
0 & TAN & Tananarive & -18.917 & 47.500 & 1.38 \\
1 & VSS & Vassouras & -22.400 & -43.650 & .50 \\
1 & PLL & Pilar & -31.667 & -63.833 & .34 \\
0 & GNA & Gnangara & -31.783 & 115.950 & .06 \\
0 & HER & Hermanus & -34.425 & 19.225 & .03 \\
1 & TOO & Toolangi & -37.533 & 145.467 & .46 \\
1 & AML & Amberley & -43.152 & 172.722 & .04 \\
\hline & & & & & \\
\hline
\end{tabular}

Code $=0$ used only for 1940-1973 analysis 1 used for both analyses (1900-1973 and 1940-1973) 2 used only for 1900-1973 analysis 


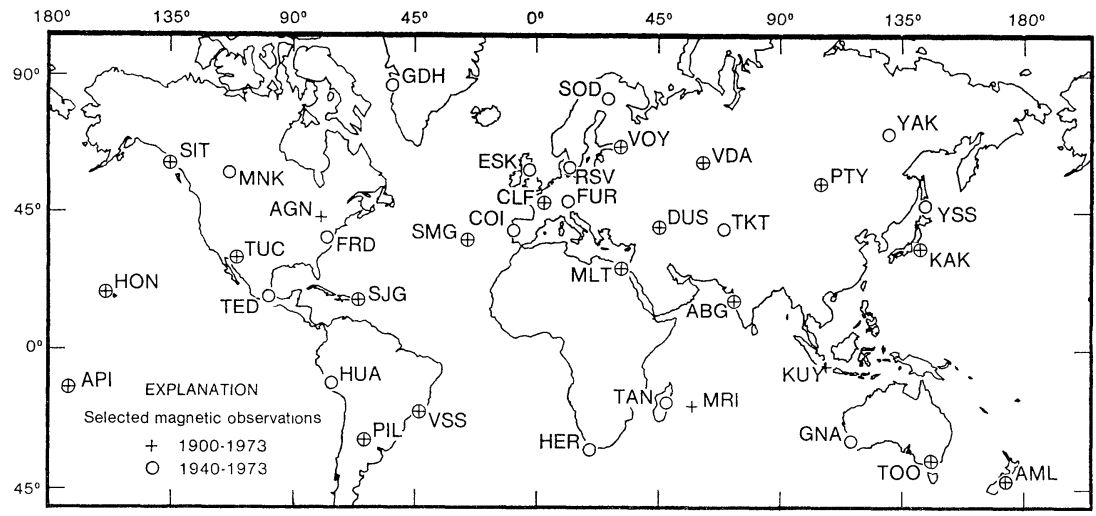

Fig. 1. Distribution of the observatories used for analysis.

The few missing data were carefully interpolated. The $X, Y$, and $Z$ components thus compiled into a homogeneous and continuous data set are listed in YukUtAKe et al. (1979). The observatories that changed their observation sites more than $3^{\circ}$ in longitude were excluded. The second set of 21 observatories from which data were similarly treated by YuKutake (1973) for the interval 1900-1965 are also indicated in Table 1 and in Fig. 1 by plus signs. These are given because the results of their earlier analysis will also be extended to 1973 and referenced later in this paper.

4. Spherical Harmonic Analysis of the Annual Mean Values of $X, Y, Z$ Components

In order to separate the variations into external and internal parts, two different methods of spherical harmonic analysis have been employed. One is the classical way that analyzes the $X$ and $Y$ components together and $Z$ separately to obtain two sets of Gauss coefficients (see e.g. Chapman and Bartels, 1940, p. 641). The internal-external separation is then made from the two sets of coefficients. The other is the analysis that treats all three components together and determines the external and the internal coefficients at once following the least squares program developed by CAIN et al. $(1965,1967)$. Since the variations from year to year are found to be essentially identical from each type of analysis only the results from the separation technique are used for interpretation. The combined analyses were made to establish confidence in the validity of the difference results and to explore the question of whether such a thin distribution of data could be used to determine absolute values of the internal and external coefficients. 
Table 2a. Gauss coefficients (nT) $\left(g_{n}^{m}(X Y), h_{n}^{m}(X Y)\right)$ obtained by analyses of the $X$ and $Y$ components. $\sigma$ represents standard deviation.

\begin{tabular}{|c|c|c|c|c|c|c|}
\hline Period & $g_{1}^{0}$ & $\sigma$ & $g_{1}^{1}$ & $\sigma$ & $h_{1}^{1}$ & $\sigma$ \\
\hline 1940 & -30403 & 221 & -2569 & 241 & 5576 & 192 \\
\hline 1941 & -30385 & 221 & -2569 & 242 & 5574 & 192 \\
\hline 1942 & -30392 & 223 & -2571 & 244 & 5575 & 194 \\
\hline 1943 & -30378 & 225 & -2563 & 246 & 5571 & 195 \\
\hline 1944 & -30378 & 225 & -2561 & 246 & 5570 & 196 \\
\hline 1945 & -30371 & 226 & -2558 & 248 & 5565 & 197 \\
\hline 1946 & -30345 & 227 & -2555 & 249 & 5557 & 197 \\
\hline 1947 & -30342 & 228 & -2546 & 251 & 5551 & 199 \\
\hline 1948 & -30339 & 230 & -2538 & 252 & 5550 & 200 \\
\hline 1949 & -30329 & 232 & -2531 & 254 & 5545 & 201 \\
\hline 1950 & -30323 & 232 & -2524 & 255 & 5543 & 202 \\
\hline 1951 & -30389 & 234 & -2517 & 257 & 5534 & 204 \\
\hline 1952 & -30300 & 236 & -2511 & 259 & 5531 & 205 \\
\hline 1953 & -30290 & 238 & -2510 & 261 & 5532 & 207 \\
\hline 1954 & -30284 & 239 & -2508 & 262 & 5534 & 208 \\
\hline 1955 & -30272 & 240 & -2503 & 262 & 5534 & 209 \\
\hline 1956 & -30247 & 240 & -2496 & 263 & 5530 & 209 \\
\hline 1957 & -30232 & 241 & -2486 & 263 & 5527 & 210 \\
\hline 1958 & -30221 & 242 & -2481 & 265 & 5524 & 211 \\
\hline 1959 & -30202 & 243 & -2478 & 266 & 5522 & 211 \\
\hline 1960 & -30178 & 243 & -2471 & 267 & 5521 & 212 \\
\hline 1961 & -30181 & 244 & -2469 & 268 & 5527 & 212 \\
\hline 1962 & -30174 & 245 & -2463 & 269 & 5529 & 213 \\
\hline 1963 & -30154 & 245 & -2455 & 269 & 5529 & 213 \\
\hline 1964 & -30141 & 246 & -2445 & 270 & 5530 & 214 \\
\hline 1965 & -30123 & 247 & -2436 & 270 & 5528 & 214 \\
\hline 1966 & -30095 & 247 & -2425 & 271 & 5521 & 215 \\
\hline 1967 & -30064 & 247 & -2410 & 271 & 5512 & 215 \\
\hline 1968 & -30041 & 248 & -2401 & 271 & 5507 & 216 \\
\hline 1969 & -30023 & 248 & -2389 & 272 & 5501 & 216 \\
\hline 1970 & -30001 & 249 & -2379 & 272 & 5491 & 216 \\
\hline 1971 & -29984 & 249 & -2369 & 272 & 5484 & 216 \\
\hline 1972 & -29959 & 249 & -2359 & 273 & 5475 & 217 \\
\hline 1973 & -29935 & 250 & -2346 & 274 & 5464 & 218 \\
\hline
\end{tabular}

\subsection{Treatment of the $X, Y$ components separate from $Z$}

Spherical harmonic analyses were made separately for the annual means from 1940 to 1973 of the $X, Y$ components and the $Z$ component of 34 observatories, assuming that all the fields are of internal origin. Thirty-four values of $Z$ component for a particular year allow a determination of the series up to the maximum degree and order of four. As is the case with spherical harmonic analysis, the resulting coefficients are dependent on the maximum degree and 
Table 2b. Gauss coefficients (nT) $\left(g_{n}^{m}(Z), h_{n}^{m}(Z)\right)$ obtained by analysis of the $Z$ component. $\sigma$ represents standard deviation.

\begin{tabular}{|c|c|c|c|c|c|c|}
\hline Period & $g_{1}^{0}$ & $\sigma$ & $g_{1}^{1}$ & $\sigma$ & $h_{1}^{1}$ & $\sigma$ \\
\hline 1940 & -31826 & 1347 & -3342 & 871 & 5952 & 932 \\
\hline 1941 & -31816 & 1351 & -3349 & 873 & 5940 & 935 \\
\hline 1942 & -31802 & 1358 & -3355 & 878 & 5928 & 940 \\
\hline 1943 & -31794 & 1364 & -3357 & 882 & 5918 & 944 \\
\hline 1944 & -31788 & 1370 & -3363 & 886 & 5912 & 949 \\
\hline 1945 & -31786 & 1378 & -3370 & 891 & 5906 & 954 \\
\hline 1946 & -31788 & 1384 & -3376 & 895 & 5895 & 958 \\
\hline 1947 & -31788 & 1392 & -3381 & 900 & 5882 & 964 \\
\hline 1948 & -31782 & 1401 & -3381 & 906 & 5880 & 969 \\
\hline 1949 & -31777 & 1408 & -3381 & 911 & 5871 & 975 \\
\hline 1950 & -31777 & 1415 & -3377 & 915 & 5869 & 979 \\
\hline 1951 & -31774 & 1421 & -3377 & 919 & 5864 & 983 \\
\hline 1952 & -31772 & 1426 & -3377 & 922 & 5855 & 987 \\
\hline 1953 & -31766 & 1431 & -3374 & 926 & 5846 & 991 \\
\hline 1954 & -31757 & 1438 & -3375 & 930 & 5840 & 995 \\
\hline 1955 & -31746 & 1441 & -3369 & 932 & 5834 & 997 \\
\hline 1956 & -31739 & 1444 & -3364 & 934 & 5825 & 999 \\
\hline 1957 & -31731 & 1448 & -3360 & 936 & 5820 & 1002 \\
\hline 1958 & -31720 & 1452 & -3354 & 938 & 5813 & 1005 \\
\hline 1959 & -31782 & 1453 & -3349 & 939 & 5809 & 1006 \\
\hline 1960 & -31688 & 1456 & -3349 & 941 & 5894 & 1008 \\
\hline 1961 & -31665 & 1458 & -3345 & 943 & 5801 & 1009 \\
\hline 1962 & -31638 & $1460^{\circ}$ & -3338 & 944 & 5798 & 1011 \\
\hline 1963 & -31620 & 1462 & -3331 & 946 & 5795 & 1012 \\
\hline 1964 & -31599 & 1463 & -3323 & 946 & 5788 & 1013 \\
\hline 1965 & -31575 & 1464 & -3315 & 947 & 5786 & 1013 \\
\hline 1966 & -31563 & 1462 & -3304 & 946 & 5786 & 1012 \\
\hline 1967 & -31549 & 1461 & -3296 & 945 & 5779 & 1011 \\
\hline 1968 & -31538 & 1459 & -3285 & 944 & 5775 & 1010 \\
\hline 1969 & -31526 & 1459 & -3274 & 943 & 5771 & 1010 \\
\hline 1970 & -31520 & 1457 & -3260 & 942 & 5770 & 1009 \\
\hline 1971 & -31504 & 1457 & -3247 & 942 & 5766 & 1008 \\
\hline 1972 & -31491 & 1454 & -3235 & 940 & 5758 & 1007 \\
\hline 1973 & -31478 & 1451 & -3223 & 938 & 5753 & 1004 \\
\hline
\end{tabular}

order at which the spherical harmonic series are terminated. The analyses were repeated, terminating the series at degrees from one to four. When only the dipole terms $n=1$ are used, long term variations in $g_{1}^{0}$ derived from the $Z$ component are different from those obtained by terminating the series at higher degrees. The approximation is evidently poor if only the dipole terms are taken. However, the variations in the coefficients with periods shorter than 20 years are similar to each other and are stable for the change in the maximum degree 
and order of the series except for the case ${ }^{\dagger} n^{*}=4$. Stability of the short-period variation was examined by taking the time derivative of each Gauss coefficient and comparing the results for different models. If the errors induced by aliasing were caused by short-period noise, they would be amplified by taking derivatives. The time-differentiated coefficients obtained from the $Z$ component with $n^{*}=4$ exhibited very different behavior with large amplitudes than did those determined from the $Z$ component with $n^{*}$ smaller than 4 . The maximum degree and order of 4 seems too large for the $34 Z$ values to give stable results. Accordingly, the Gauss coefficients determined up to $n^{*}=3$ were adopted for the analysis of the $Z$ component and were combined with those of $n^{*}=4$ for the $(X, Y)$ analysis to separate the internal field from the external field. Gauss coefficients obtained separately from the $X$ and $Y$ analysis $\left(g_{n}^{m}(X Y), h_{n}^{n}(X Y)\right)$ and from the $Z$ analysis $\left(g_{n}^{m}(Z), h_{n}^{m}(Z)\right)$ for the $n=1$ terms are shown in Table $2 \mathrm{a}$ and $\mathrm{b}$.

The standard deviations given in Table $2 \mathrm{a}$ and $\mathrm{b}$ are very large. They range from $221 \mathrm{nT}$ to $250 \mathrm{nT}$ for $g_{1}^{0}(X Y)$. Due to the large size of the standard deviation it might appear meaningless to discuss a year-to-year change in the dipole component that is less than $30 \mathrm{nT} / \mathrm{yr}$ for $g_{1}^{0}(X Y)$. However, the variability of the year-to-year change in the magnetic components from one observatory to another is not so large as might be suspected from this large standard deviation for the main field. Taking the year-to-year variation of the observed field as $\Delta X, \Delta Y$, and $\Delta Z$, we repeated the spherical harmonic analysis for $\Delta X$ and $\Delta Y$ separately from $\Delta Z$. The Gauss coefficients thus obtained, e.g. $\Delta g_{1}^{0}(X Y)$, were the same as those obtained by simply taking the annual differences of the coefficients in Table $2 \mathrm{a}$ and $\mathrm{b}$. The standard deviations for $\Delta g_{1}^{0}(X Y)$ were much reduced, ranging from $2.4 \mathrm{nT} / \mathrm{yr}$ to $3.6 \mathrm{nT} / \mathrm{yr}$. This implies that the year-to-year variations in the Gauss coefficients in Table $2 \mathrm{a}$ and $\mathrm{b}$ are well above the range of scatter of the rate of change in the geomagnetic components at the individual observatories. Therefore, in spite of the large standard deviations of the Gauss coefficients for the main field, the relative yearly change in the coefficients is regarded as real and useful for examination of the time change in the dipole component.

Representation by a spherical harmonic series terminated at such low degrees as $n=3$ or 4 is only a crude approximation of the geomagnetic field. The number of the observatories used for the analysis is also very limited. If there are a few observatories whose fields deviate largely from the smooth field represented by the low-degree harmonics, the standard deviations assigned to each coefficient are expected to become large. At some of the observatories deviations from the smooth field indeed exceed 1,000 nT. Even though small fluctua-

$t$ The convention used here, suggested by R. Hide, is to express the use of the whole series up to $n=m$ by the notation $n^{*}$. 
tions such as the solar cycle variations are superposed, the major part of the deviations remains nearly constant during a period of several decades. Removal of this constant part of the deviation from the original data will greatly reduce the standard deviations of the Gauss coefficients for the main field.

The effect of the number of observatories was examined by reducing the number from 34 to 20 . Observatories with relatively noisy $Z$ variations were intentionally excluded. However, no improvement was observed by this procedure. Indeed, the scatter of the results had a tendency to be slightly greater.

Table 3. External and internal Gauss coefficients (nT) computed from $\left(g_{n}^{m}(X Y)\right.$, $\left.h_{n}^{m}(X Y)\right)$ and $\left(g_{n}^{m}(Z), h_{n}^{m}(Z)\right)$.

\begin{tabular}{|c|c|c|c|c|c|c|}
\hline Year & $g_{1}^{0 e}$ & $g_{1}^{0 i}$ & $g_{1}^{1 e}$ & $h_{1}^{1 e}$ & $g_{1}^{1 i}$ & $h_{1}^{1 i}$ \\
\hline 1940 & 949 & -31352 & 515 & -251 & -3084 & 5827 \\
\hline 1941 & 954 & -31339 & 520 & -244 & -3089 & 5818 \\
\hline 1942 & 940 & -31332 & 523 & -235 & -3094 & 5810 \\
\hline 1943 & 944 & -31322 & 529 & -231 & -3092 & 5802 \\
\hline 1944 & 940 & -31318 & 535 & -228 & -3096 & 5798 \\
\hline 1945 & 943 & -31314 & 541 & -227 & -3099 & 5792 \\
\hline 1946 & 962 & -31307 & 547 & -225 & -3102 & 5782 \\
\hline 1947 & 964 & -31306 & 557 & -221 & -3103 & 5772 \\
\hline 1948 & 962 & -31301 & 562 & -220 & -3100 & 5770 \\
\hline 1949 & 965 & -31294 & 567 & -217 & -3098 & 5762 \\
\hline 1950 & 969 & -31292 & 569 & -217 & -3093 & 5760 \\
\hline 1951 & 977 & -31286 & 573 & -220 & -3090 & 5754 \\
\hline 1952 & 981 & -31281 & 577 & -216 & -3088 & 5747 \\
\hline 1953 & 980 & -31270 & 576 & -209 & -3086 & 5741 \\
\hline 1954 & 982 & -31266 & 578 & -204 & -3086 & 5738 \\
\hline 1955 & 983 & -31255 & 577 & -200 & -3080 & 5734 \\
\hline 1956 & 995 & -31242 & 579 & -197 & -3075 & 5727 \\
\hline 1957 & 999 & -31231 & 583 & -195 & -3069 & 5722 \\
\hline 1958 & 999 & -31220 & 582 & -193 & -3063 & 5717 \\
\hline 1959 & 1000 & -31202 & 581 & -191 & -3059 & 5713 \\
\hline 1960 & 1007 & -31185 & 585 & -189 & -3056 & 5710 \\
\hline 1961 & 989 & -31173 & 584 & -183 & -3053 & 5710 \\
\hline 1962 & 976 & -31159 & 583 & -179 & -3046 & 5708 \\
\hline 1963 & 977 & -31131 & 584 & -177 & -3039 & 5706 \\
\hline 1964 & 972 & -31113 & 585 & -172 & -3030 & 5702 \\
\hline 1965 & 968 & -31091 & 586 & -172 & -3022 & 5700 \\
\hline 1966 & 979 & -31074 & 586 & -177 & -3011 & 5698 \\
\hline 1967 & 990 & -31054 & 591 & -178 & -3001 & 5690 \\
\hline 1968 & 998 & -31039 & 589 & -179 & -2990 & 5686 \\
\hline 1969 & 1002 & -31025 & 590 & -182 & -2979 & 5681 \\
\hline 1970 & 1013 & -31014 & 587 & -186 & -2966 & 5677 \\
\hline 1971 & 1013 & -30997 & 585 & -188 & -2954 & 5672 \\
\hline 1972 & 1021 & -30980 & 584 & -189 & -2943 & 5664 \\
\hline 1973 & 1029 & -30964 & 585 & -193 & -2931 & 5657 \\
\hline
\end{tabular}


If the observed variations originated entirely inside the earth (and there were no contamination from neglected higher order coefficients), then the terms $\left(g_{n}^{m}(X Y), h_{n}^{m}(X Y)\right)$ and $\left(g_{n}^{m}(Z), h_{n}^{m}(Z)\right)$ in Table $2 \mathrm{a}$ and b should show complete agreement with each other. The discrepancies between these terms, except for the part due to contamination, indicate the existence of geomagnetic variations of external origin. The separation into external $\left(g_{n}^{m e}, h_{n}^{m e}\right)$ and internal $\left(g_{n}^{m i}, h_{n}^{m i}\right)$ terms up to $n^{*}=3$ were made by the following formula:

$$
\begin{array}{ll}
g_{n}^{m e}=\frac{(n+1) g_{n}^{m}(X Y)-(n+1) g_{n}^{m}(Z)}{2 n+1}, & g_{n}^{m i}=\frac{n g_{n}^{m}(X Y)+(n+1) g_{n}^{m}(Z)}{2 n+1}, \\
h_{n}^{m e}=\frac{(n+1) h_{n}^{m}(X Y)-(n+1) h_{n}^{m}(Z)}{2 n+1}, & h_{n}^{m i}=\frac{n h_{n}^{m}(X Y)+(n+1) h_{n}^{m}(Z)}{2 n+1} .
\end{array}
$$

As seen in the above equations, the separation process depends on the difference between the horizontal and vertical solutions. Since there are twice as many points in the $X Y$ solution, the $Z$ determination would contribute more noise even if it were as accurately observed as either $X$ or $Y$. However, baseline maintenance is the hardest for $Z$, and errors most easily enter this component. It is not very certain whether the data available at present, particularly those for the $Z$ component, are accurate enough to permit us to extend the separation up to the higher harmonics. Accordingly, only the results for the first-degree
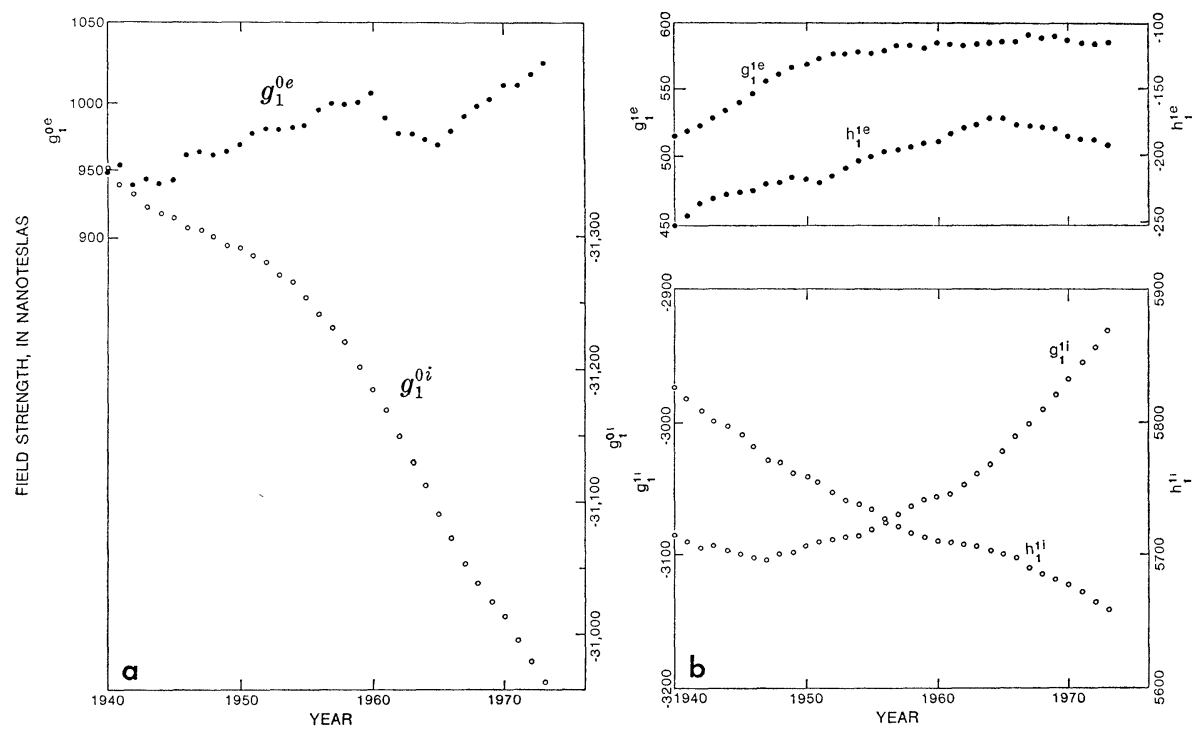

Fig. 2. a. Time variations in the external and internal parts of the axial dipole component $\left(g_{1}^{0}\right)$. b. Time variations in the external and internal parts of the equatorial dipole component $\left(g_{1}^{1}, h_{1}^{1}\right)$. Unit in $\mathrm{nT}$. 
terms are shown in Table 3. If the contamination of the results is due to the neglected terms, it should be relatively constant since the changes in field are small compared to its magnitude. Thus, as will be shown later, one can rely on the changes revealed by this analysis, but not on the absolute values themselves. Figure 2 shows the time variations in the external and internal parts of the first-degree terms. In contrast to the smooth changes in the internal coefficients, zigzag features are noticeable in the external coefficients.

\subsection{Determination of the external coefficients simultaneously from three components}

Since the direct method of analysis using the classical component separation technique gave such large residuals and significant biases in the absolute results, some other techniques were applied to attempt to improve the analysis and to investigate the statistical validity of the variations discussed. These other numerical experiments will be reported on here, but are not used extensively in the interpretations, because they were only partially successful and the results were basically identical to those by the previous technique. However, as will be shown subsequently, they do indicate the difficulty in obtaining absolute values from the selected observatories and of using statistical evidence to either validate or refute the previous results.

The alternate method of computation is based on the technique developed by CAIN et al. $(1965,1967)$, by which a first-order Taylor's expansion is performed to linearize the least-squares solution for fitting any observed component of the magnetic field. Since the components chosen for this study were $X, Y$, and $Z$ annual means, a non-linear solution was not actually required, but experience has shown that a second iteration very slightly improves the solution, and the same least-squares matrix must be constructed anyway to compute the standard errors and cross-correlation coefficients. The program takes into account the Earth's oblateness and the varying altitudes of the observatories. The oblateness correction was originally shown (CAIN et al., 1965) to be important for obtaining a more coherent organization of the data. That is, even a remapping of the data back onto a spheroid by ignoring oblateness in both the analysis and recomputation of the field was shown to cause distortions in the components as great as $150 \mathrm{nT}$ near the Earth's surface. Since the separation technique ignored oblateness, a numerical experiment was performed to test the effect on the external field computations. $X, Y$, and $Z$ values of the Earth's field were first computed using the IGRF model at epoch 1965 up to $n^{*}=4$ on a $10^{\circ}$ by $10^{\circ}$ grid of latitude and longitude on the spheroidal earth. The resulting vectors were then analyzed by the field model program altered to set $F_{\theta}=-X, F_{r}=-Z$, and $a / r=1$. That is, the geocentric components of field $F$ expressed in spherical coordinates $\left(F_{\theta}\right.$ and $\left.F_{r}\right)$ differ slightly in direction from the geodetically observed 
$X$ and $Z$, and the geocentric radius also varies with latitude from the standard value " $a$ " normally taken in geomagnetic computations to be about $6,371 \mathrm{~km}$ (corresponding to the Earth's mean radius). Using an $n^{*}=4$ solution plus the first external term, the following rms residuals were obtained:

$\begin{array}{cc}\text { Component } & \text { rms (nT) } \\ X & 52 \\ Y & 28 \\ Z & 75 \\ \text { All } & 55\end{array}$

False external coefficients of $g_{1}^{0}=-82.2 \mathrm{nT}, g_{1}^{1}=3.1 \mathrm{nT}$, and $h_{1}^{1}=-7.7 \mathrm{nT}$ were generated. The standard deviation of the coefficients was $\pm 1.8 \mathrm{nT}$ and they had only small cross correlations with any of the internal terms.

It is thus clear that while a large spurious external component is created by neglecting oblateness, its size is still an order of magnitude smaller than that seen in the separation analysis. Since any absolute variations in dipole position over the epochs analyzed are very small, we could also assume its effect constant.

A second test was then performed to determine the extent to which spurious external components can be created by perturbations in the data, both on a uniform grid and at the positions of the selected observatories. In the latter case it was observed that adding gaussian noise whose variance was $10 \mathrm{nT}$ to each component and fitting data generated from an $n^{*}=4$ internal model to a model that had the same internal complexity and had first-order externals, produced spurious external components no larger than $0.5 \mathrm{nT}$. These externals were statistically insignificant and equally distributed among the three coefficients.

The third experiment was to test for aliasing from neglected higher order terms by creating data on an equal grid from an internal model of terms $n^{*}=8$ but fitting the data only with $n^{*}=4$ internal and $n=1$ external coefficients. This procedure gave much larger false externals that were statistically insignificant. From this result it is clear that neglecting higher order terms is the basic cause for biases like those found in the results from the separation technique. These previous results were then duplicated (except that a consistent $n^{*}$ was used) by analyzing the 1940-1973 selected data from the 34 observatories with a series limited to only $n^{*}=4$ internal and $n=1$ external terms. This computation gave rms residuals to the data from 750 to $813 \mathrm{nT}$, standard errors of the resulting coefficients from 50 to $200 \mathrm{nT}$, and external terms parallel to the equator $\left(g_{1}^{1}\right.$ and $h_{1}^{1}$ external) as much as several hundred nanoteslas. The low-order terms were inspected and seen to exhibit year-to-year variations very similar to those produced by the separation analysis. 
We then addressed the question of whether it would be possible to obtain accurate absolute values of the low-order field components using the data from 34 observatories.

The technique attempted was to use information other than that provided by the selected observatories to reduce the contamination. As mentioned earlier the spacing between the selected points (as much as $10,000 \mathrm{~km}$ ) was so great that determinations of coefficients beyond $n^{*}=4$ would not be reliable. The

Table 4a. Average deviations of 1940-1973 annual means from the GSFC(12/66) field model (nT).

\begin{tabular}{|c|c|c|c|}
\hline & $X$ & $Y$ & $Z$ \\
\hline Godhavn & 265 & -245 & 480 \\
\hline Sodankylä & -210 & -66 & -553 \\
\hline Yakutsk & 0 & -1231 & 192 \\
\hline Voyeykovo & 44 & 73 & -203 \\
\hline Sitka & 6 & 9 & -90 \\
\hline Vysokaya-Dubrava & -305 & -124 & -414 \\
\hline Meanook & 88 & -2 & -140 \\
\hline Rude Skov & 22 & 26 & -2 \\
\hline Eskdalemuir & -18 & -20 & -40 \\
\hline Patrony & 9 & 64 & 6 \\
\hline Fürstenfeldbruck & -16 & 17 & 15 \\
\hline Chambon-la-Forêt & -61 & -2 & 133 \\
\hline Yuzhno Sakhalinsk & 8 & -166 & -131 \\
\hline Dusheti & -236 & 45 & -49 \\
\hline Tashkent & -271 & 37 & -93 \\
\hline Coimbra & 33 & -15 & -75 \\
\hline Fredericksburg & -3 & -83 & 67 \\
\hline San Miguel & 675 & 431 & 1667 \\
\hline Kakioka & -6 & -7 & -95 \\
\hline Tucson & -142 & -61 & 158 \\
\hline Misallat & -39 & 65 & 100 \\
\hline Honolulu & -142 & 80 & -416 \\
\hline Teoloyucan & -82 & 25 & 19 \\
\hline Alibag & -119 & 464 & 580 \\
\hline San Juan & -183 & 178 & 252 \\
\hline Huancayo & 119 & 116 & 117 \\
\hline Apia & -123 & 224 & -938 \\
\hline Tananarive & 291 & 11 & -385 \\
\hline Vassouras & 54 & -55 & -22 \\
\hline Pilar & 83 & -3 & -24 \\
\hline Gnangara & -78 & -85 & 135 \\
\hline Hermanus & -37 & 9 & 38 \\
\hline Toolangi & -10 & -82 & 84 \\
\hline Amberley & 16 & -30 & 5 \\
\hline
\end{tabular}


data were corrected for the higher order terms of the field by use of the GSFC(12/66) field model (CAIN et al., 1967). Since the intent was only to test the technique, no extensive comparisons were made with results from other models. Of course the GSFC model could only correct for field complexity up to its limit of $n^{*}=10$, while it is known (e.g. REGAN et al., 1975) that there are significant components of much higher degree and order extending to the very small scale local anomalies. However, if we assume that most of this upper part of the spatial spectrum is crustal and thus constant, a further noise reduction is possible by computing the average difference over the time span of the data between the field model and each observatory component. Table 4a gives the average deviation between the model field and each component for each observatory; these deviations were used to correct each component prior to analysis. Thus the analysis was performed on the data after the values in Table 4 a were subtracted. Only the $n=1$ external and $n^{*}=4$ internal terms were allowed to vary; whereas the $n=5$ to 10 terms of the model were used to further correct the data. The results of this analysis for the $n=1$ external terms are
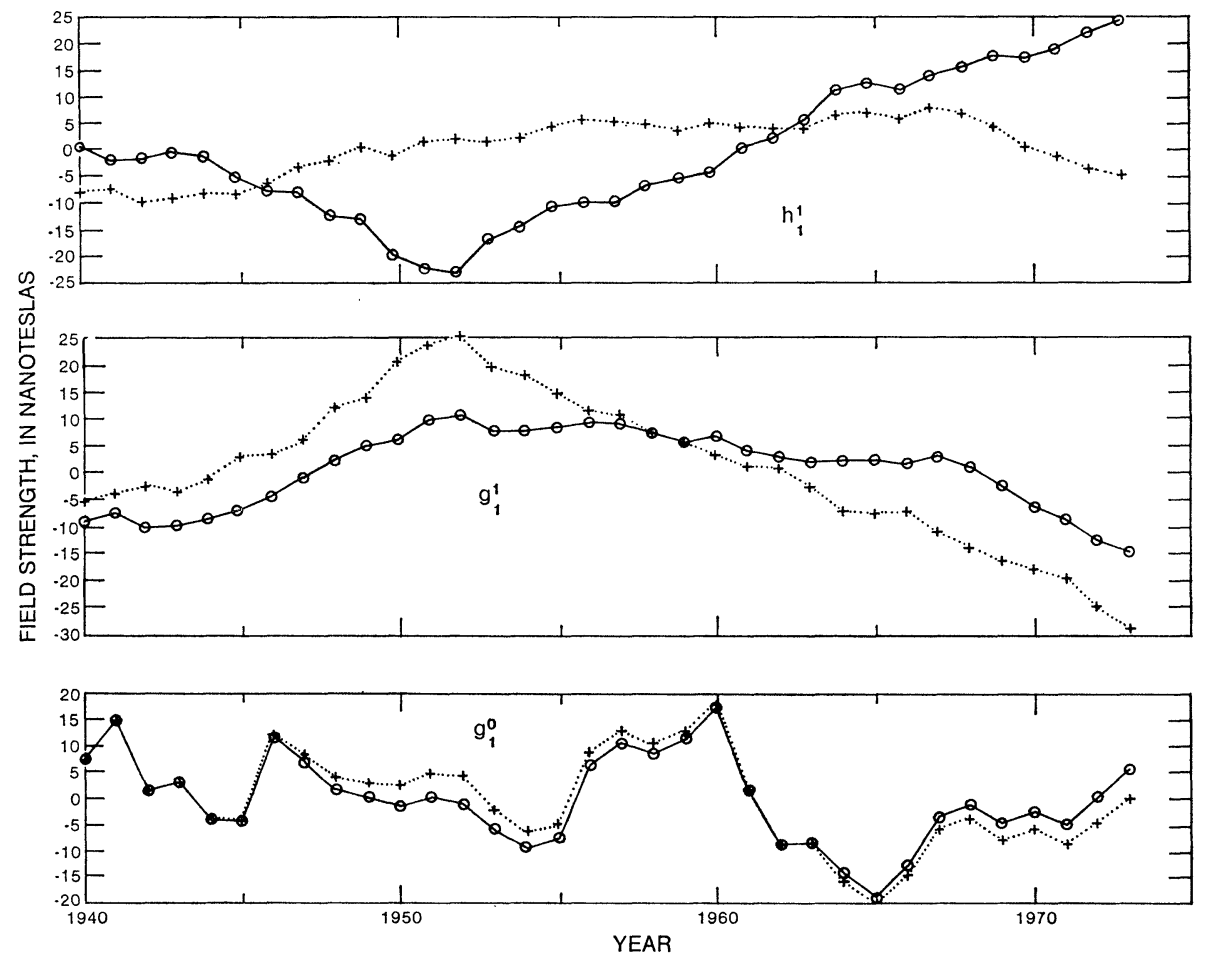

Fig. 3. First-degree external coefficients obtained by one step analysis to $n^{*}=4$ of annual means precorrected for higher degree internal terms. 
illustrated by the solid lines in Fig. 3, and a tabulation for both internal and external terms for $n=1$ is given in Table $4 \mathrm{~b}$. The limitation to $n=1$ in the external components is based on past experience (CAIN and DAvIs, 1973) that the higher order components are relatively small.

Table 4b. External and internal coefficients (nT) resulting from analysis of 34 station annual means after correction for GSFC(12/66) field model terms above $n=4$. Adjustments made to coefficients up to $n=4$ correcting data for first and second coefficients of GSFC(12/66) model from $n=5$ through $n=10$.

\begin{tabular}{|c|c|c|c|c|c|c|}
\hline \multirow{2}{*}{ Year } & \multicolumn{3}{|c|}{ External terms } & \multicolumn{3}{|c|}{ Internal terms } \\
\hline & $g_{1}^{0}$ & $g_{1}^{1}$ & $h_{1}^{1}$ & $g_{1}^{0}$ & $g_{1}^{1}$ & $h_{1}^{1}$ \\
\hline 1940 & 8.2 & -8.7 & .6 & -30343.6 & -2155.6 & 5751.9 \\
\hline 1941 & 15.1 & -7.2 & -2.0 & -30351.1 & -2157.5 & 5758.8 \\
\hline 1942 & 1.9 & -10.0 & -1.7 & -30361.3 & -2157.0 & 5765.5 \\
\hline 1943 & 3.7 & -9.6 & -.8 & -30367.8 & -2155.6 & 5767.1 \\
\hline 1944 & -3.6 & -8.5 & -1.4 & -30378.0 & -2156.9 & 5772.7 \\
\hline 1945 & -3.9 & -7.0 & -5.3 & -30387.2 & -2158.3 & 5777.0 \\
\hline 1946 & 11.5 & -4.3 & -7.9 & -30392.1 & -2159.9 & 5777.0 \\
\hline 1947 & 7.2 & -.6 & -8.4 & -30402.9 & -2159.9 & 5777.6 \\
\hline 1948 & 2.0 & 2.4 & -12.7 & -30410.4 & -2157.9 & 5782.9 \\
\hline 1949 & .6 & 5.1 & -13.4 & -30415.8 & -2157.6 & 5782.5 \\
\hline 1950 & -1.1 & 6.1 & -20.0 & -30423.7 & -2156.1 & 5789.1 \\
\hline 1951 & .6 & 9.9 & -22.3 & -30429.1 & -2158.6 & 5787.7 \\
\hline 1952 & -.4 & 10.7 & -23.4 & -30435.9 & -2159.0 & 5789.4 \\
\hline 1953 & -5.4 & 7.7 & -17.2 & -30437.8 & -2159.7 & 5788.5 \\
\hline 1954 & -9.3 & 7.8 & -14.7 & -30443.4 & -2161.9 & 5791.4 \\
\hline 1955 & -7.2 & 8.6 & -11.2 & -30443.3 & -2162.3 & 5791.8 \\
\hline 1956 & 7.0 & 9.5 & -10.3 & -30446.6 & -2162.2 & 5789.5 \\
\hline 1957 & 10.9 & 9.1 & -10.1 & -30448.2 & -2160.7 & 5790.7 \\
\hline 1958 & 9.2 & 7.5 & -7.3 & -30449.5 & -2162.4 & 5788.7 \\
\hline 1959 & 12.0 & 5.8 & -5.8 & -30446.7 & -2163.3 & 5788.9 \\
\hline 1960 & 17.4 & 6.7 & -4.7 & -30442.0 & -2166.5 & 5790.1 \\
\hline 1961 & 2.1 & 4.2 & -.1 & -30441.9 & -2170.0 & 5793.4 \\
\hline 1962 & -8.5 & 3.1 & 1.8 & -30436.1 & -2169.9 & 5796.6 \\
\hline 1963 & -8.2 & 2.0 & 5.2 & -30429.3 & -2171.0 & 5796.5 \\
\hline 1964 & -14.1 & 2.4 & 11.3 & -30422.7 & -2171.3 & 5793.9 \\
\hline 1965 & -18.5 & 2.5 & 12.7 & -30412.0 & -2170.2 & 5792.5 \\
\hline 1966 & -12.9 & 1.8 & 11.1 & -30403.2 & -2170.4 & 5789.7 \\
\hline 1967 & -3.3 & 3.1 & 13.8 & -30394.2 & -2171.7 & 5783.3 \\
\hline 1968 & -1.0 & 1.3 & 15.6 & -30386.7 & -2172.9 & 5778.5 \\
\hline 1969 & -4.7 & -2.2 & 17.8 & -30379.1 & -2172.1 & 5772.2 \\
\hline 1970 & -2.4 & -6.1 & 17.5 & -30372.6 & -2172.0 & 5765.7 \\
\hline 1971 & -4.9 & -8.5 & 19.0 & -30364.7 & -2170.2 & 5758.7 \\
\hline 1972 & .5 & -12.4 & 22.3 & -30356.0 & -2169.8 & 5748.2 \\
\hline 1973 & 5.6 & -14.6 & 24.9 & -30347.9 & -2170.0 & 5736.7 \\
\hline
\end{tabular}


The validity of these results can be examined in the light of the previously mentioned tests on the synthetic data and the statistics from the analysis. Table $4 \mathrm{c}$ presents the residuals (averaged to 5-year intervals), standard errors, $\sigma_{p}$, and $t=p / \sigma_{p}$ (Student's " $t$ " test); $p$ is the magnitude of the total external field vector (CAIN et al., 1965). These averages were taken from the results for each year simply for convenience in reducing the size of the table, not because there were any large deviations for a given year. Although the standard errors are taken from those determined for magnitude of the external field vector, they are comparable to those for all of the components. Values of 1.96 and 0.67 for $t$ refer to confidence levels of $95 \%$ and $50 \%$ respectively. The cross-correlation between the parameters was also examined and found to be very low.

The second technique used to investigate the validity of these results was to take successive differences ("secular variation") of the annual means, and to separately analyze these changes. This approximation was considered appropriate since the non-linear secular change terms are insignificant over a time span as short as a year.

In making the analysis of secular change the same computer code was employed, but the $\dot{X}, \dot{Y}, \dot{Z}$ data replaced $X, Y, Z$, and the GSFC $(12 / 66)$ coefficients $\dot{g}, \dot{h}, 2 \ddot{g}, 2 \ddot{h}, 0,0$ replaced $g, h, \dot{g}, \dot{h}, \ddot{g}, \ddot{h}$ respectively. The solution was then for the potential $\dot{V}$ in place of $V$, and only the $\dot{g}$ and $\dot{h}$ terms were adjusted.

The results of this difference analysis are seen to be approximately equivalent to those from the combined analyses. This analysis was done with adjustments in the linear secular change terms up to $n^{*}=4$ and in the externals, (now also representing changes of field) allowing both linear and quadratic secular terms of the GSFC(12/66) model to continue in effect (no equivalent "anomaly" correction) from $n=5$ to 10 . The coefficients of secular change from 1941 through 1973 are given in Table 4d. Here of course, since the previous dates referred to the middle of the year (e.g. 1940.5), the first differences are more correctly assigned to the epoch at the end (e.g. 1941.0).

Table 4c. Statistics from analyses of annual means averaged over five year intervals.

\begin{tabular}{|c|c|c|c|c|c|c|}
\hline \multirow{2}{*}{ Years } & \multicolumn{4}{|c|}{ Residuals (nT) } & \multicolumn{2}{|l|}{ External fields } \\
\hline & $X$ & $Y$ & $Z$ & All & Standard error $(\mathrm{nT})$ & $t$ \\
\hline $1940-1944$ & 37 & 43 & 77 & 55 & 12 & 1.0 \\
\hline 1945-1949 & 36 & 36 & 59 & 45 & 9 & 1.4 \\
\hline $1950-1954$ & 39 & 23 & 44 & 37 & 7 & 2.9 \\
\hline $1955-1959$ & 33 & 16 & 28 & 28 & 6 & 2.7 \\
\hline $1960-1964$ & 24 & 21 & 32 & 26 & 5 & 2.3 \\
\hline $1965-1969$ & 37 & 36 & 59 & 46 & 9 & 2.0 \\
\hline $1970-1973$ & 82 & 59 & 100 & 82 & 14 & 1.4 \\
\hline
\end{tabular}


The residuals of fit to the various components over the interval are similar in pattern to those of Table $4 \mathrm{c}$, but the absolute values are smaller. They average from 7 to $9 \mathrm{nT} / \mathrm{yr}$ from 1941 through 1966 and rise to $12 \mathrm{nT} / \mathrm{yr}$ by 1973, the increase being due to the errors in the higher order correction terms since the field model was constructed with data only to 1965 . The $\dot{Y}$ component contributes very little since its residuals are almost always below those for $\dot{X}$ and $\dot{Z}$. The residuals in $\dot{Z}$ were not commonly higher than those in $\dot{X}$, implying

Table 4d. External and internal coefficients (nT) of annual change based on annual mean differences from 34 observatories.

\begin{tabular}{|c|c|c|c|c|c|c|c|}
\hline \multirow{2}{*}{ Year } & \multicolumn{3}{|c|}{ External coefficients } & \multicolumn{4}{|c|}{ Internal coefficients } \\
\hline & $\dot{g}_{1}^{0}$ & $\dot{g}_{1}^{1}$ & $\dot{h}_{1}^{1}$ & $\dot{g}_{1}^{0}$ & $\dot{g}_{1}^{1}$ & $\dot{h}_{1}^{1}$ & $\dot{g}_{2}^{0}$ \\
\hline 1941 & 8.4 & 1.9 & -1.8 & 7.2 & 4.9 & 2.1 & -20.2 \\
\hline 1942 & -11.7 & -3.1 & .9 & 4.4 & 8.0 & 1.8 & -21.5 \\
\hline 1943 & 3.2 & .2 & 1.6 & 7.9 & 8.9 & -3.0 & -23.3 \\
\hline 1944 & -5.9 & 1.1 & .1 & 4.3 & 6.3 & .9 & -21.8 \\
\hline 1945 & .9 & 1.8 & -3.6 & 5.2 & 6.6 & .0 & -19.9 \\
\hline 1946 & 17.2 & 2.6 & -2.3 & 9.3 & 6.2 & -4.6 & -21.1 \\
\hline 1947 & -3.4 & 3.5 & .1 & 3.6 & 7.8 & -3.8 & -18.9 \\
\hline 1948 & -4.3 & 2.1 & -2.8 & 7.3 & 10.3 & .2 & -24.2 \\
\hline 1949 & .1 & 2.5 & -.5 & 8.6 & 8.3 & -4.7 & -20.7 \\
\hline 1950 & -.3 & .8 & -6.3 & 6.5 & 9.7 & 2.7 & -18.9 \\
\hline 1951 & 2.0 & 3.2 & -2.1 & 8.9 & 5.2 & -5.6 & -21.1 \\
\hline 1952 & -.3 & .8 & -.8 & 7.5 & 7.8 & -2.5 & -21.1 \\
\hline 1953 & -4.1 & -3.1 & 6.4 & 12.4 & 7.7 & -4.8 & -21.9 \\
\hline 1954 & -3.3 & .2 & 3.0 & 8.8 & 6.1 & -1.6 & -20.1 \\
\hline 1955 & 2.5 & .7 & 3.7 & 12.3 & 8.1 & -3.6 & -20.9 \\
\hline 1956 & 14.6 & 1.2 & 1.7 & 12.8 & 7.8 & -7.1 & -19.6 \\
\hline 1957 & 4.2 & -.5 & .3 & 12.5 & 10.0 & -2.6 & -20.4 \\
\hline 1958 & -1.7 & -2.1 & 2.9 & 12.2 & 7.3 & -6.0 & -22.3 \\
\hline 1959 & 2.9 & -1.5 & 1.4 & 16.8 & 7.9 & -3.6 & -19.1 \\
\hline 1960 & 5.5 & 1.1 & 1.1 & 18.7 & 5.7 & -2.4 & -22.9 \\
\hline 1961 & -15.9 & -2.4 & 4.6 & 14.5 & 5.3 & -.2 & -22.4 \\
\hline 1962 & -10.7 & -1.2 & 1.7 & 19.8 & 8.9 & -.5 & -21.3 \\
\hline 1963 & -.1 & -1.1 & 3.1 & 20.7 & 7.9 & -3.8 & -21.7 \\
\hline 1964 & -6.1 & .5 & 6.0 & 20.6 & 8.7 & -6.1 & -19.8 \\
\hline 1965 & -5.2 & .5 & 1.7 & 25.2 & 10.0 & -4.9 & -23.2 \\
\hline 1966 & 5.0 & -1.0 & -1.7 & 22.8 & 9.7 & -6.6 & -17.9 \\
\hline 1967 & 8.4 & 1.6 & 1.6 & 23.2 & 7.6 & -8.7 & -19.7 \\
\hline 1968 & 1.6 & -1.9 & 1.3 & 21.3 & 8.1 & -8.1 & -19.1 \\
\hline 1969 & -4.3 & -2.6 & 3.5 & 22.2 & 10.4 & -10.9 & -20.5 \\
\hline 1970 & 1.4 & -4.3 & -.8 & 20.3 & 9.5 & -9.6 & -19.4 \\
\hline 1971 & -3.3 & -1.6 & 2.3 & 22.3 & 11.6 & -10.9 & -19.2 \\
\hline 1972 & 4.3 & -4.3 & 2.5 & 22.4 & 10.1 & -13.3 & -19.0 \\
\hline 1973 & 3.8 & -.5 & 3.0 & 21.6 & 9.2 & -15.6 & -19.5 \\
\hline
\end{tabular}

Adjustments made to coefficients up to $n=4$ correcting data for first and second order coefficients of GSFC(12/66) model from $n=5$ through $n=10$. 
that there was no evidence for a higher observational error in this component. The estimated standard errors of the resulting external coefficients range from 1.9 to $1.4 \mathrm{nT} / \mathrm{yr}$ from 1941 to 1965 and rise to $2.7 \mathrm{nT} / \mathrm{yr}$ by 1973 .

In reviewing these results to evaluate the amount of confidence we may have in either the coefficients of the absolute determination or the differences, we are struck by the fact that the statistics themselves are insufficient. That is, even though the standard errors of the absolute determinations using the combined method on the reduced data are satisfyingly low, the resulting internal coefficients differ significantly from those determined from more complete data sets. For example, a comparison of the low-order terms for 1965 from Table $4 \mathrm{~b}$ with those of two other models from 1975 is as follows:

\begin{tabular}{crcc} 
Internal & \multicolumn{3}{c}{ Model (data in nT) } \\
\hline Coefficient & Table 4b & GSFC $(12 / 66)$ & IGS75 \\
$g_{1}^{0}$ & -30412 & -30333 & -30337 \\
$g_{1}^{1}$ & -2170 & -2117 & -2117 \\
$h_{1}^{1}$ & 5792 & 5759 & 5759
\end{tabular}

Since the GSFC and IGS75 (BARRACLOUGH et al., 1975) models were derived without regard to any possible external field, one might ask whether they might be in error instead of the values from Table $4 \mathrm{~b}$. A numerical experiment was performed to determine the effect of a known external field on such determinations. The IGRF model (CAIN and CAIN, 1971) was used to create a uniform grid of data to which was then added an external field parallel to the dipole of $50 \mathrm{nT}$. The resulting vectors were then analyzed up to $n^{*}=4$ without allowing the external coefficients to vary. The largest change was to $g_{1}^{0}$ and was only $7.6 \mathrm{nT}$. The other coefficients were altered by $1 \mathrm{nT}$ or less. It is concluded that although the statistics indicate that the coefficients from the combined analysis of corrected data (both internal and external) are in error only of the order of $10 \mathrm{nT}$ (Table 4c) it is likely that they could be biased as much as $70 \mathrm{nT}$ from the true values.

A similar comparison is tabulated for the secular change results as follows (for epoch 1970 in $\mathrm{nT} / \mathrm{yr}$ ):

\begin{tabular}{ccccc} 
Internal & \multicolumn{4}{c}{ Model } \\
\hline Coefficient & Table 4d & GSFC(12/66) & AWC75 & IGS75 \\
$g_{1}^{0}$ & 20 & 13 & 25 & 23 \\
$g_{1}^{1}$ & 10 & 11 & 10 & 10 \\
$h_{1}^{1}$ & -10 & -0 & -10 & -10 \\
$g_{2}^{0}$ & -19 & -26 & -25 & -24
\end{tabular}

$\uparrow$ Peddie and Fabiano (1976). 
This table appears to indicate that the $2-3 \mathrm{nT} / \mathrm{yr}$ error estimates on the secular change coefficients are not unreasonable when the variability of the values in Table $4 \mathrm{~d}$ is taken into account. That is, one cannot argue that these biases are large compared to those from other models, as was the case for the absolute determinations. It would appear that standard errors of the order of $2 \mathrm{nT} / \mathrm{yr}$ in the year-to-year changes are not unreasonable.

\section{Double Peaks in $g_{1}^{0 e}$ Variations during a Solar Cycle}

From the time variations in the dipole components shown in Fig. 2a, general trends, approximated by a parabolic equation for $g_{1}^{0 e}$ and a third degree polynomial for $g_{1}^{0 i}$, were subtracted, and the results are shown in Fig. 4. In the $g_{1}^{0 e}$ variation a peak appears in 1960 , and then $g_{1}^{0 e}$ decreases rapidly, reaching a minimum in 1965 . This peak-to-trough variation amounts to about $47 \mathrm{nT}$. Besides this large amplitude variation, small fluctuations with peaks at intervals of several years are noticeable. Regarding the plot of $g_{1}^{0 i}$, a phenomenon having a period of about 20 years is so predominant that any changes with shorter periods are hard to see in this diagram. Then a high-pass filter was used to extract shorter period variations, and the results are illustrated in Fig. 5.

There is seen in Fig. 4 a relatively large variation in the internal parts of the equatorial dipole component. The amplitudes of $g_{1}^{1 i}$ and $h_{1}^{1 i}$ are nearly the same as those of $g_{1}^{1 e}$ and $h_{1}^{1 e}$. From induction theory such a large variation in the internal terms is unlikely to be induced by the change in the corresponding external component. The maximum amount theoretically expected for the equatorial dipole on a homogeneous perfect conductor model is 50 percent of the inducing field (see, e.g. Chapman and BARTeLs, 1940, pp. 732-749). If the fluctuation of the equatorial dipole shown in Fig. 4 is real, and if this is due to induction, we must consider such mechanisms as lateral inhomogeneities within the earth. Another possible interpretation is to regard this relatively large variation as a phenomenon originating from inside the core. As will be discussed later, variations of internal origin of the axial dipole for periods less than 10 years can be regarded mostly as effects of the field induced by the external variation. Therefore, at the present stage it seems rather difficult to ascribe this internal equatorial dipole variation to a core origin.

Residuals after removing the general trend were passed through a numerical high-pass filter with its cut off period around 10 years. The characteristics of this 11 point-filter were such that periods over 20 years were reduced to less than $2 \%$ and those of 15 years $24 \%$, whereas periods under 10 years remained between 92 and $110 \%$ of the original amplitude. Variations symmetric to the end points were added before and after the data window. The high-passed 

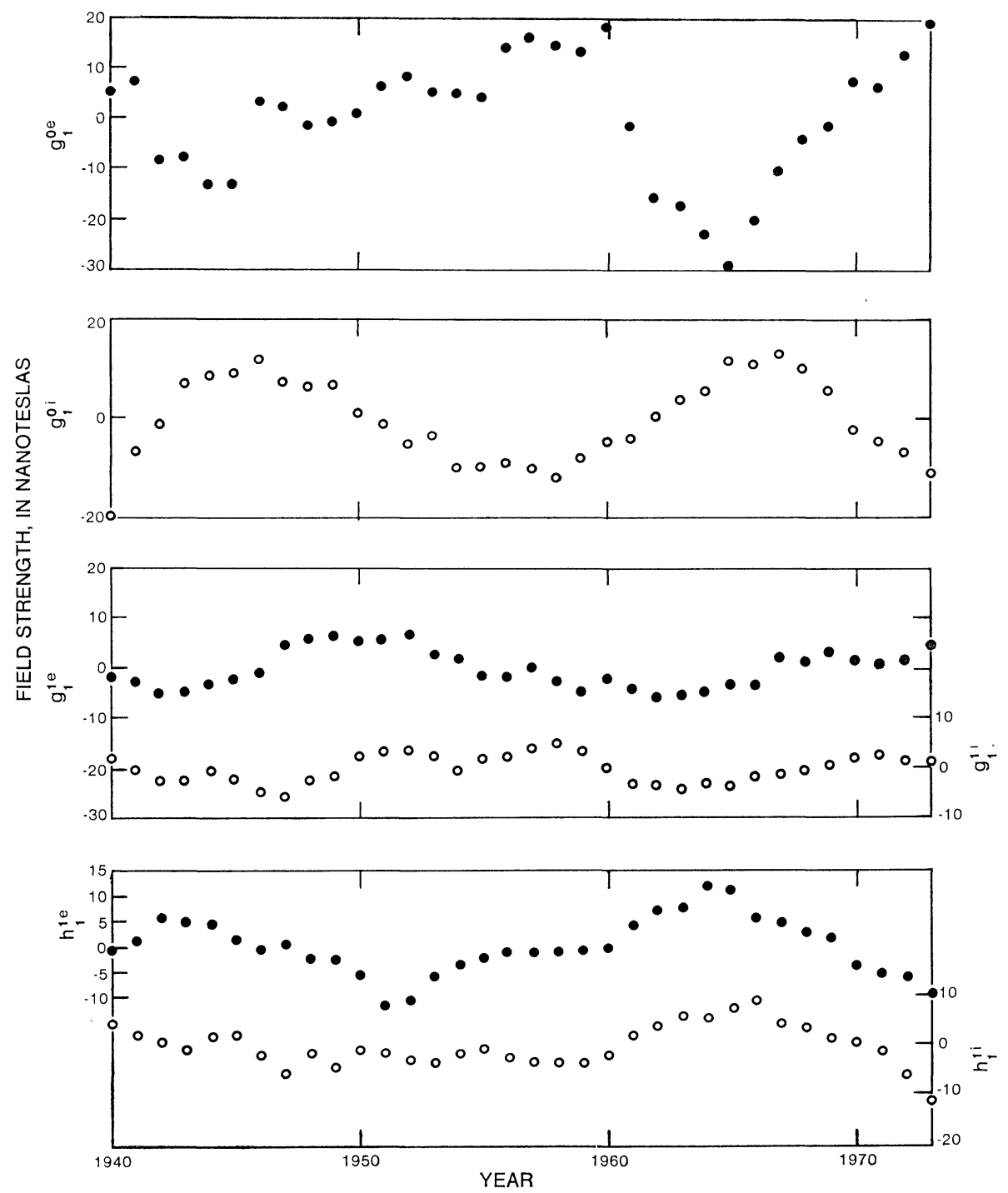

Fig. 4. Time variations in the dipole terms after trend removal.

results for $g_{1}^{0 e}$ and $g_{1}^{0 i}$ are shown in Fig. 5 in comparison with time variations in some other phenomena. From the top down, the figure shows the relative sunspot numbers, the annual number of days when $A p$ indices exceeded 60 (Meyers and Allen, 1977), and the annual Dst values (Sugiura and Poros, 1977).

One of the remarkable features revealed by a straightforward comparison 

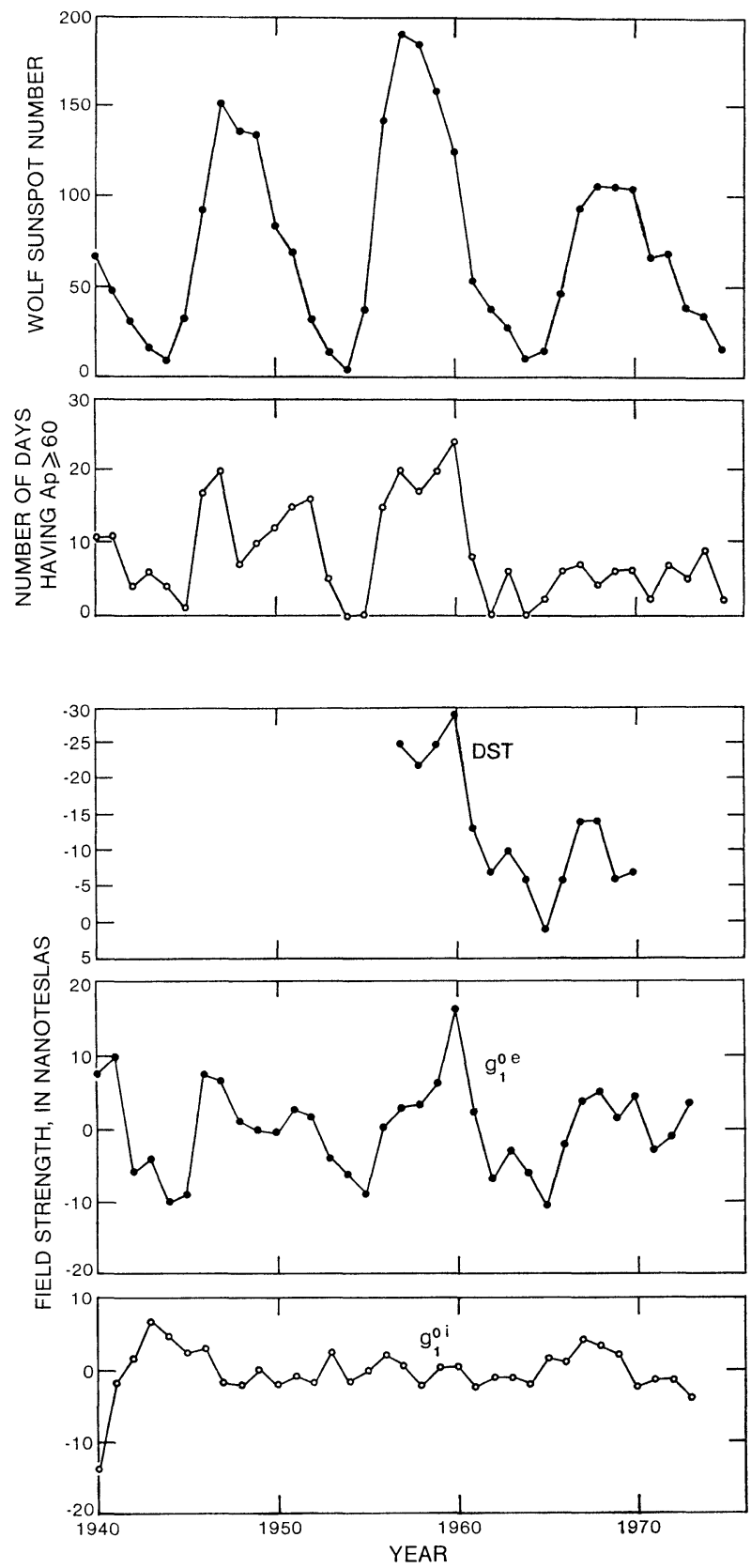

Fig. 5. Comparison of variations in the axial dipole field with those of other geomagnetic indices. From the top down are shown the annual values of the relative sunspot number, number of days when $A p$ index exceeded 60, annual Dst values, and the axial dipole components of external and the internal origin after being passed through a high-pass filter. 
of $g_{1}^{0 e}$ with the relative sunspot number in Fig. 5 is the rather poor correspondence of their year-to-year variations, although minima of the sunspot number coincide with those of $g_{1}^{0 e}$. A peak in $g_{1}^{0 e}$ appears in 1946, preceeding the maximum of the sunspot number. The next peak in $g_{1}^{0 e}$ corresponds to a decreasing period of the sunspot number. In a general sense, $g_{1}^{0 e}$ changes with the same cycle as the sunspot numbers, but two peaks appear in the change of $g_{1}^{0 e}$ during each solar cycle.

This phenomenon is also seen in other geomagnetic parameters. Ap indices, shown in Fig. 5, have two peaks in a solar cycle. Dst values show the same tendency. Among these geomagnetic quantities almost complete correspondence exists from year to year. Correlation coefficients are very high between these quantities, as will be discussed in the following section. These results are almost identical with those obtained by ALLDREDGE et al. (1978) for recent observatory data.

Another aspect we should note here is a small variation in $g_{1}^{0 i}$. No significant variation is seen during the period from 1947 to 1964.

\section{The External Field along the Geomagnetic Axis}

Variations of the external coefficients $g_{1}^{0 e}, g_{1}^{1 e}$, and $h_{1}^{1 e}$ (Fig. 4) were passed through a numerical band pass filter that has a frequency characteristic with its peak around a 10-year period. The results are shown in Fig. 6 by open circles. It can be seen that changes in the equatorial components $g_{1}^{1 e}$ and $h_{1}^{1 e}$ closely correspond to those of the axial component $g_{1}^{0 e}$. The quantity $g_{1}^{1 e}$ changes in phase with $g_{1}^{0 e}$, whereas $h_{1}^{1 e}$ is completely out of phase. If the ratios of the equatorial coefficients $\left(g_{1}^{1 e}\right.$ and $\left.h_{1}^{1 e}\right)$ to the axial one $\left(g_{1}^{0 e}\right)$ are the same as the ratios of the main field that specify the inclined angle of the geomagnetic axis, the direction of the variation exactly parallels the inclined axis. This relationship is partly satisfied in the present case, i.e. regarding the phase. When $g_{1}^{0 e}$ changes in a negative direction, $g_{1}^{1 e}$ changes in the same direction but $h_{1}^{1 e}$ changes in a positive direction. On the other hand, the main field coefficients $g_{1}^{0}$ and $g_{1}^{1}$ are both negative, while $h_{1}^{1}$ is positive. Therefore the variations represented by the first degree terms in Fig. 4 must be approximately aligned in a direction parallel to the inclined geomagnetic axis.

The coordinate system was thus rotated from the geographic coordinates to those in which one of the axis coincides with that of the internal dipole. Assuming a boreal dipole position of $79^{\circ} \mathrm{N}$ and $69^{\circ} \mathrm{W}$, the coefficients may be transformed by the expressions

$$
G_{1}^{0}=g_{1}^{0} \cos 11^{\circ}+Q \sin 11^{\circ} \quad \text { where } Q=g_{1}^{1} \cos 69^{\circ}-h_{1}^{1} \sin 69^{\circ},
$$




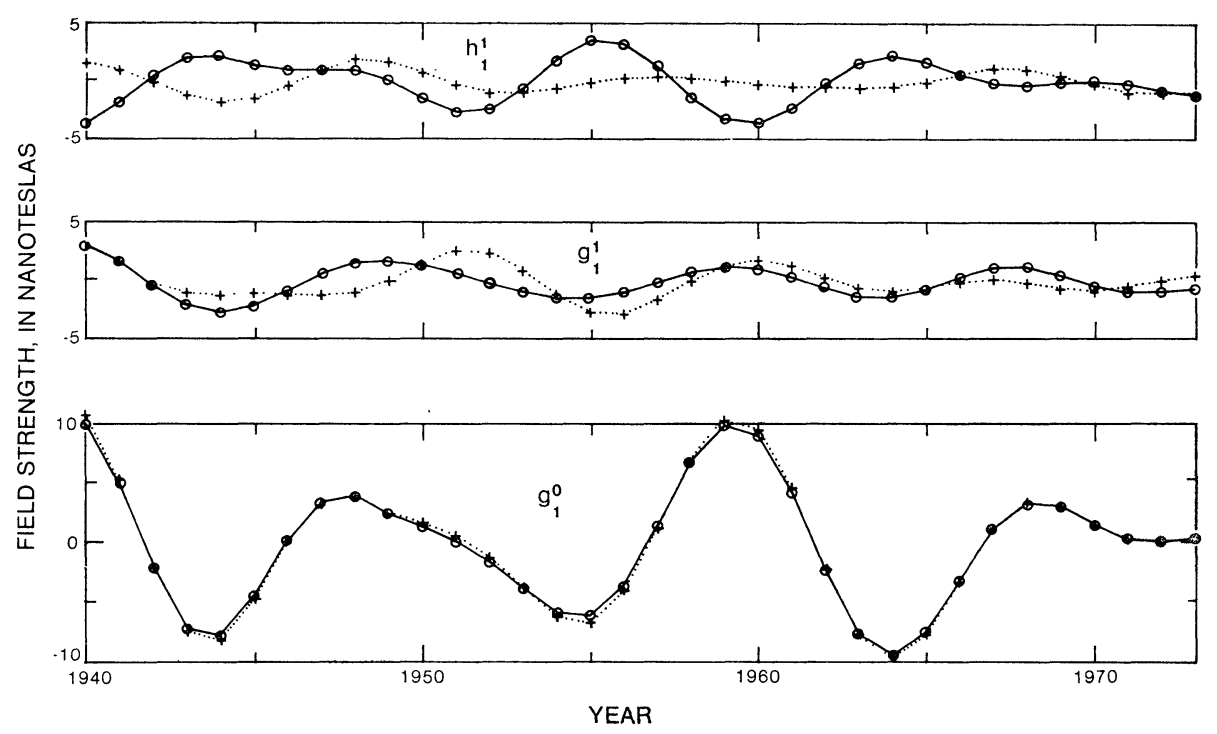

Fig. 6. The values $g_{1}^{0}, g_{1}^{1}, h_{1}^{1}$ are passed through a numerical band-pass filter with a peak around a 10-year period. Open circles are the filtered results. + signs show variations for the new coordinates, one of whose axis is taken along the geomagnetic axis. Reduction of the amplitude by the rotation of the coordinates is seen for $h_{1}^{1}$ and $g_{1}^{\mathbf{1}}$.

$$
\begin{aligned}
& G_{1}^{1}=Q \cos 11^{\circ}-g_{1}^{0} \sin 11^{\circ}, \quad \text { and } \\
& H_{1}^{1}=h_{1}^{1} \cos 69^{\circ}+g_{1}^{1} \sin 69^{\circ} .
\end{aligned}
$$

Since the secular changes are all linear, the above expressions may also be applied to the $\dot{g}$ and $\dot{h}$ terms.

The variations obtained by this transformation are shown by the dotted curves in Fig. 6. The variation along the geomagnetic axis is the largest and preserves the main feature of $g_{1}^{0 e}$. Reduction of the amplitudes of the orthogonal components is obvious particularly in $h_{1}^{1 e}$. Our previous qualitative conclusion that the solar cycle variation is approximately aligned along the geomagnetic axis rather than along the geographic axis is thus further strengthened.

The results obtained by the combined analyses in Tables $4 \mathrm{~b}$ and $4 \mathrm{~d}$ were also transformed into the magnetic coordinates by the above rotation expressions and are illustrated by the dotted curves in Fig. 3. Then, the relations of the rotated and non rotated components to other geophysical indices were examined. The first-order externals from Fig. 6 were correlated with the number of days per year whose $A p$ index exceeded a specified level (Meyers and Allen, 1977), with the Wolf sunspot number, and with annual average Dst values. These results are illustrated in Table $4 \mathrm{e}$. It is clear from this table that variation of 
Table 4e. Statistical comparisons between external field results and geophysical parameters (SPSS package used, NiE et $a l, 1975$ ).

\begin{tabular}{|c|c|c|c|c|c|c|c|c|c|c|}
\hline & \multicolumn{9}{|c|}{ Pearson correlation coefficients } \\
\hline & & Dst & $A p>40$ & Sunspots & $g_{1}^{0}$ & $G_{1}^{0}$ & $g_{1}^{1}$ & $G_{1}^{1}$ & $h_{1}^{1}$ & $H_{1}^{1}$ \\
\hline \multirow{9}{*}{ 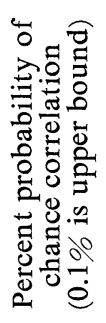 } & Dst & - & -.82 & -.75 & -.95 & -.92 & -.13 & -.23 & .41 & .22 \\
\hline & $A p>40$ & $.1 \%$ & - & .63 & .93 & .94 & .29 & .42 & -.58 & -.12 \\
\hline & Sunspots & $.1 \%$ & $.3 \%$ & - & .75 & .75 & .40 & .44 & -.55 & .10 \\
\hline & $g_{1}^{0}$ & $.1 \%$ & $.1 \%$ & $.1 \%$ & - & .98 & .17 & .31 & -.50 & -.24 \\
\hline & $G_{1}^{0}$ & $.1 \%$ & $.1 \%$ & $.1 \%$ & $.1 \%$ & - & .35 & .49 & -.66 & -.10 \\
\hline & $g_{1}^{1}$ & $31 . \%$ & $13 . \%$ & $6 . \%$ & $25 . \%$ & 8. $\%$ & - & .94 & -.86 & -.83 \\
\hline & $G_{1}^{1}$ & $18 . \%$ & 5. $\%$ & 4. $\%$ & $11 . \%$ & 2. $\%$ & $.1 \%$ & - & -.98 & .60 \\
\hline & $h_{1}^{1}$ & 5. $\%$ & $.7 \%$ & 1. \% & 2. $\%$ & $.2 \%$ & $.1 \%$ & $.1 \%$ & - & -.44 \\
\hline & $H_{1}^{1}$ & $20 . \%$ & $32 . \%$ & $34 . \%$ & $18 . \%$ & $36 . \%$ & $.1 \%$ & $.5 \%$ & $4 . \%$ & - \\
\hline
\end{tabular}

the external values obtained are the results of magnetic activity as manifested by the changes in average Dst*. It is equally clear that the rotation of coordinates to the dipole completely eliminates the weak correlation of the $h_{1}^{1}$ coefficient with Dst, while neither $g_{1}^{1}$ nor $G_{1}^{1}$ are significantly correlated. A similar correlation was done between the secular change coefficients and the successive differences of Dst and gave the following results:

Correlation coefficient with Dst

$\begin{array}{lr}\dot{g}_{1}^{0} & -.98 \\ \dot{G}_{1}^{0} & -.90 \\ \dot{g}_{1}^{1} & -.11 \\ \dot{G}_{1}^{1} & -.07 \\ \dot{h}_{1}^{1} & .67 \\ \dot{H}_{1}^{1} & .15\end{array}$

Probability of chance correlarion (.1\% is upper bound)

$$
\begin{aligned}
& .1 \% \\
& .1 \% \\
& 35 \% \\
& 40 \% \\
& .2 \% \\
& 28 \%
\end{aligned}
$$

Although the correlation with the tilted dipole is not quite as high as for the "absolute" analysis, both of the terms perpendicular to the dipole are satisfyingly uncorrelated.

Both of these results would confirm our previous conclusions that the yearto-year external variations are indeed real. However, the biases noted earlier for the internal absolute values would indicate the possibility also of similar biases (as large as a few tens of $\mathrm{nT}$ ) in the external values as well.

The mean $G_{1}^{0}$ for $1957-1973$ is $-3 \mathrm{nT}$, whereas that for Dst $=-14 \mathrm{nT}$, implying that $G_{1}^{0}$ is $-17 \mathrm{nT}$ when Dst $=0 * *$. The results given by CAIN (1966) on

* For background information on the Peasson correlation coefficients see NiE et al. (1975, p. 280).

** Note that $G_{1}^{0}$ is positive when the external field is downward on the boreal (north) pole whereas Dst is positive when the field is northward. 
the average external field for the interval 1945-1964 can be rotated to give a value for $G_{1}^{0}$ of $-21 \mathrm{nT}$, implying that the quiet day value is fairly small. However, M. Sugiura (private communication, 1977) believes on the basis of analysis of satellite data that Dst $=0$ when $G_{1}^{0}=+40 \mathrm{nT}$. Also, if the all-day curves of FoRBUSH (1964) are correct, then the absolute $G_{1}^{0}$ determined herein would be some $40 \mathrm{nT}$ too large. It would appear that further work is indicated before these discrepancies can be resolved.

\section{Double Solar Cycle}

It is still a point of argument whether or not the "22-year" variation exists in the geomagnetic field in association with the polarity change in the sunspot

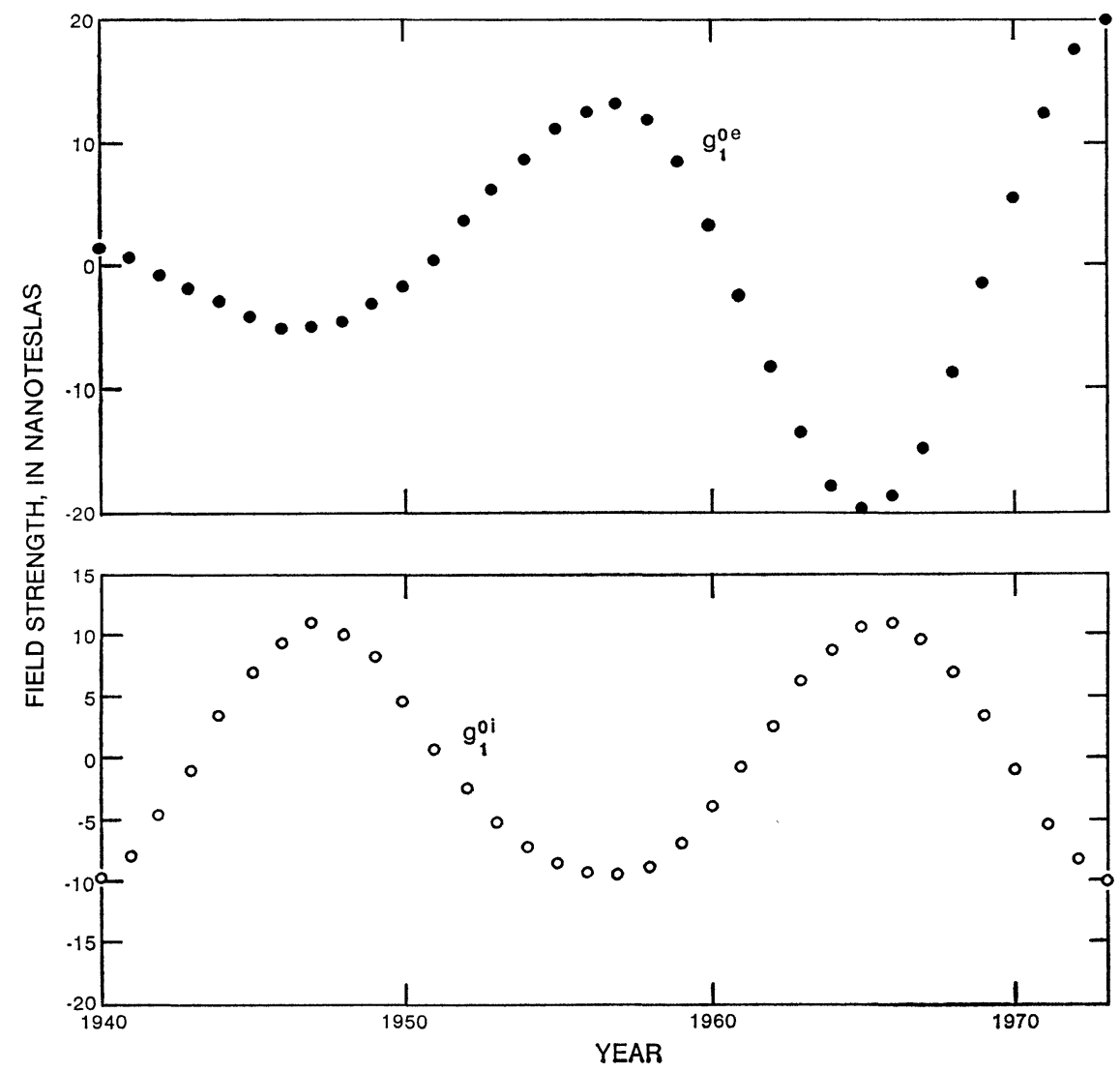

Fig. 7. Variations in the dipole $\left(g_{1}^{0 e}, g_{1}^{0 i}\right)$ when they are subjected to a numerical band pass filter with a peak around 20 years. 
field. CURRIE (1973) reported a 22-year periodicity in the observatory data, but COURTILLOT and LEMOUËL (1976) cast a serious doubt about its significance, and Alldredge (1977) considered that variations having a period of about 20 years were of internal origin.

To the axial dipole components $g_{1}^{0 e}$ and $g_{1}^{0 i}$ in Fig. 4, we applied a numerical band pass filter with its peak at about a 20 -year period. The results are shown in Fig. 7. In the external variation $g_{1}^{0 e}$, the interval from one peak to another is 18 years. This is not the period of the pure double solar cycle. A large variation amounting to $33 \mathrm{nT}$ from peak to trough is seen between 1957 and 1965 . This trend is obviously recognizable in the unfiltered variation in Figs. 3 and 4. The effects of the solar cycle on the geomagnetic field are not identical from one cycle to another. This could be one cause of long-term variations in the external field in Fig. 7, though it is uncertain if this is the only cause or not.

In the internal variation $g_{1}^{0 i}$, a more periodic variation amounting to $21 \mathrm{nT}$ from peak to trough was obtained. The period is 19 years. The ratio of the internal to the external coefficient becomes larger than 0.6. The cause of such a high ratio is unknown.

Although the present analysis does not succeed in settling debate on the double solar cycle variation, the possibility cannot be ignored that considerable contribution to this period range comes from inside the core. A further analysis will be needed to derive conclusive results.

\section{Solar Cycle Variation in the First-Degree Components since 1900}

The axial dipole component in the analysis of data from 21 observatories that cover the period from 1900 to 1973 (YUKUTAKE, 1973) was examined to extract the internal parts of the solar cycle variation. A few of these observatories were not included in the previous set of 34 observatories as shown in Fig. 1.

The quality of data is poorer in the older records. Perhaps because of the difficulty in accurate maintenance of base-line values, the amplitude of the solar cycle variation in the dipole coefficient obtained from the $Z$ component is unreasonably large for the first few decades of this century, compared with that from the $X$ and $Y$ components, though the phases of the variation look real. Therefore, a weighting function to reduce the large amplitude was adopted.

The dipole components obtained separately from $(X, Y)$ and $Z$ were first passed through a numerical high-pass filter that has its cut off frequency around a 10 -year period. The variations obtained from the $Z$ components were unreasonably large for the earlier part of the data set, whereas those from $(X, Y)$ were nearly stationary for the whole period. Since it appeared that the early 
$Z$ variation increase was merely due to the higher inaccuracies of measurement prior to 1940 , a weighting function was constructed to minimize its effect. Then the internal field variations were separated from the external changes. The weighting function adopted was of the form $w_{i}=1-a t_{i}^{\prime 2}$, where $t_{i}^{\prime}=t_{i}-1973$, since the "noise" appeared to increase parabolically to 1900 . The $a$ was tentatively determined to minimize the sum square of either (1) the external variation, or (2) the internal variation. In both cases, however, use of the weighting function gave such a ridiculous result that $w_{i}$ became negative at the beginning of the data series (though the results were nearly the same except for the epochs when the weight was negative). Since such a negative weight is physically meaningless, $a$ was simply determined so as to make $w_{i}$ zero at 1900 . The results did not differ very much by these three different treatments, except for the internal variations before 1910 .
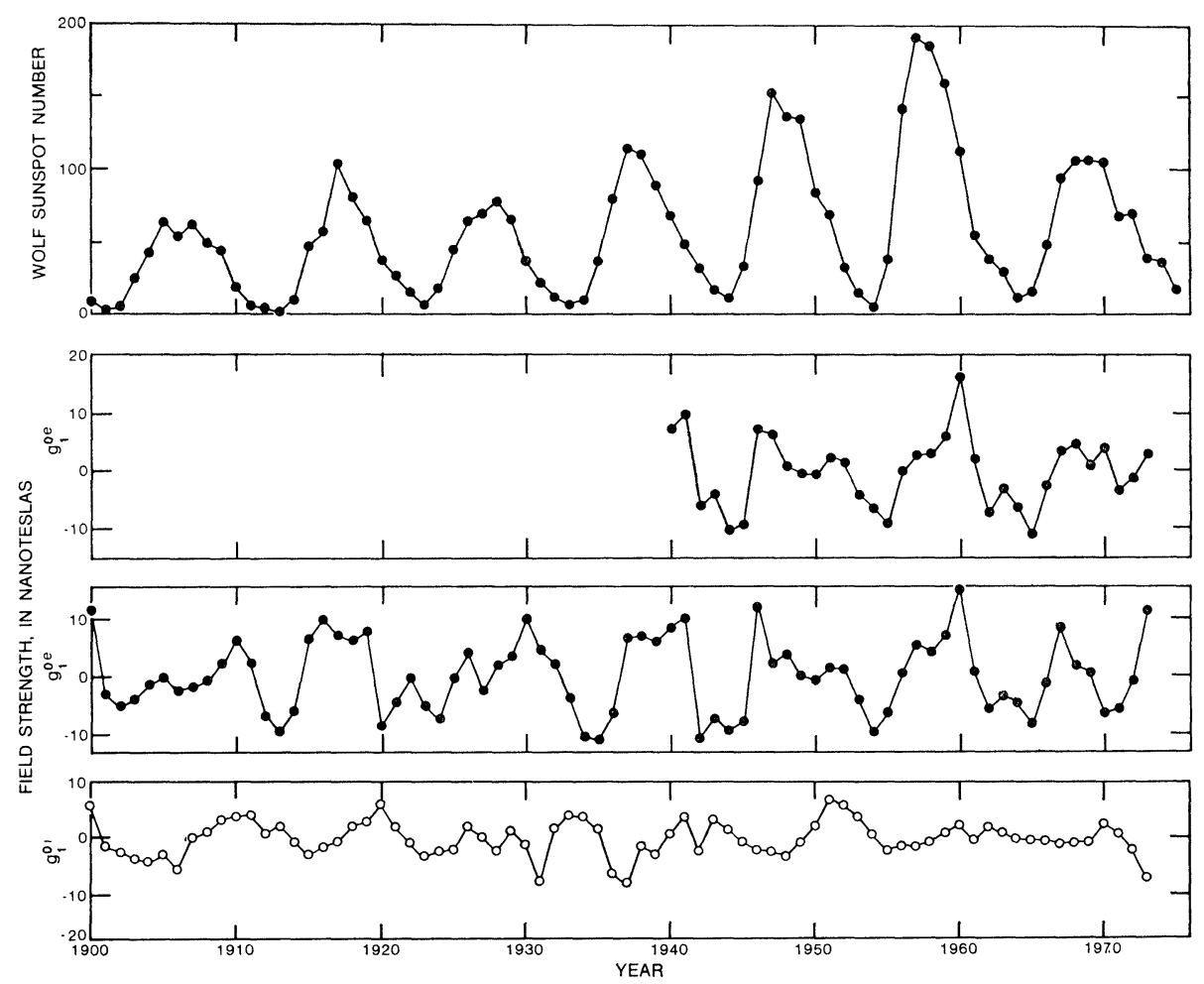

Fig. 8. Solar cycle variations in the axial dipole component from 1900 to 1973 . From the top down are shown relative sunspot numbers, the external variations $\left(g_{1}^{D_{e}}\right)$ obtained from 34 observatory data, the external variations $\left(g_{1}^{0 e}\right)$ obtained from 21 observatory data, and the internal variations $\left(g_{1}^{0 i}\right)$ in the axial dipole component. 
Table 5. External and internal coefficients (nT) of the first order terms for the period from 1900 to 1973 after being passed through a high pass filter.

\begin{tabular}{|c|c|c|c|c|c|}
\hline Year & External & Internal & Year & External & Internal \\
\hline 1900 & 11.6 & 5.8 & 1937 & 6.4 & -7.8 \\
\hline 1901 & -2.8 & -1.2 & 1938 & 7.0 & -1.0 \\
\hline 1902 & -5.1 & -2.3 & 1939 & 5.8 & -2.7 \\
\hline 1903 & -4.0 & -3.6 & 1940 & 8.4 & .9 \\
\hline 1904 & -1.4 & -4.0 & 1941 & 10.0 & 3.8 \\
\hline 1905 & -.2 & -2.9 & 1942 & -11.0 & -2.3 \\
\hline 1906 & -2.4 & -5.3 & 1943 & -7.3 & 3.4 \\
\hline 1907 & -2.0 & .3 & 1944 & -9.5 & 1.7 \\
\hline 1908 & -.7 & 1.1 & 1945 & -7.8 & -.4 \\
\hline 1909 & 2.2 & 3.5 & 1946 & 11.7 & -2.0 \\
\hline 1910 & 6.4 & 3.9 & 1947 & 2.2 & -2.3 \\
\hline 1911 & 2.3 & 3.9 & 1948 & 3.7 & -3.0 \\
\hline 1912 & -6.8 & .9 & 1949 & -.1 & -.5 \\
\hline 1913 & -9.6 & 2.3 & 1950 & -.9 & 2.2 \\
\hline 1914 & -5.8 & -.5 & 1951 & 1.3 & 7.1 \\
\hline 1915 & 6.5 & -2.9 & 1952 & 1.2 & 5.8 \\
\hline 1916 & 9.8 & -1.6 & 1953 & -4.2 & 3.8 \\
\hline 1917 & 7.2 & -.4 & 1954 & -9.7 & .6 \\
\hline 1918 & 6.1 & 2.3 & 1955 & -6.4 & -2.2 \\
\hline 1919 & 7.7 & 2.8 & 1956 & .4 & -1.3 \\
\hline 1920 & -8.4 & 6.1 & 1957 & 5.4 & -1.5 \\
\hline 1921 & -4.6 & 2.1 & 1958 & 4.0 & -.6 \\
\hline 1922 & -.3 & -.8 & 1959 & 7.0 & 1.2 \\
\hline 1923 & -5.0 & -3.1 & 1960 & 14.7 & 2.4 \\
\hline 1924 & -7.2 & -2.4 & 1961 & .5 & -.3 \\
\hline 1925 & -.4 & -2.1 & 1962 & -5.7 & 2.0 \\
\hline 1926 & 4.1 & 2.4 & 1963 & -3.7 & 1.0 \\
\hline 1927 & -2.5 & .4 & 1964 & -4.8 & -.2 \\
\hline 1928 & 1.9 & -2.2 & 1965 & -8.6 & -.4 \\
\hline 1929 & 3.3 & 1.5 & 1966 & -1.4 & -.4 \\
\hline 1930 & 10.0 & -1.2 & 1967 & 8.4 & -.8 \\
\hline 1931 & 4.5 & -7.6 & 1968 & 1.6 & -.8 \\
\hline 1932 & 2.0 & 1.9 & 1969 & .4 & -.7 \\
\hline 1933 & -4.0 & 4.2 & 1970 & -6.7 & 2.7 \\
\hline 1934 & -10.4 & 2.9 & 1971 & -5.9 & .9 \\
\hline 1935 & -11.2 & 1.6 & 1972 & -1.1 & -2.1 \\
\hline 1936 & -6.4 & -6.2 & 1973 & 11.6 & -6.8 \\
\hline
\end{tabular}

The external $\left(g_{1}^{0 e}\right)$ and the internal $\left(g_{1}^{0 i}\right)$ components thus obtained are plotted in Fig. 8 and listed in Table 5. The external coefficients obtained for the latest data set, i.e. 34 observatory data from 1940 to 1973, are also shown for comparison. Except for peaks in 1946 and 1967 for the 21-observatory data set, the variations in $g_{1}^{0 e}$ are almost identical between the two data sets. 
It is evident that the variation in $g_{1}^{0 e}$ is not parallel with that of the relative sunspot number. Almost without exception, double peaks appear in $g_{1}^{0 e}$ during one solar cycle. Mostly, the first peak appears either at the same time as the maximum of the sunspot number or one year before it. The second peak is at the declining stage of solar activity. This relation can be seen more clearly in Fig. 9, where $g_{1}^{0 e}, g_{1}^{0 i}$, and the annual values of the relative sunspot number were picked up at an interval of 10 years for 50-year period and were averaged. It is clear from this figure that, on an average, epochs of minimum $g_{1}^{0 e}$ correspond to those of the sunspot number.

The variation in the internal coefficient $g_{1}^{0 i}$ looks very noisy in Fig. 8. However, when the annual values are superposed as in Fig. 9, at the interval
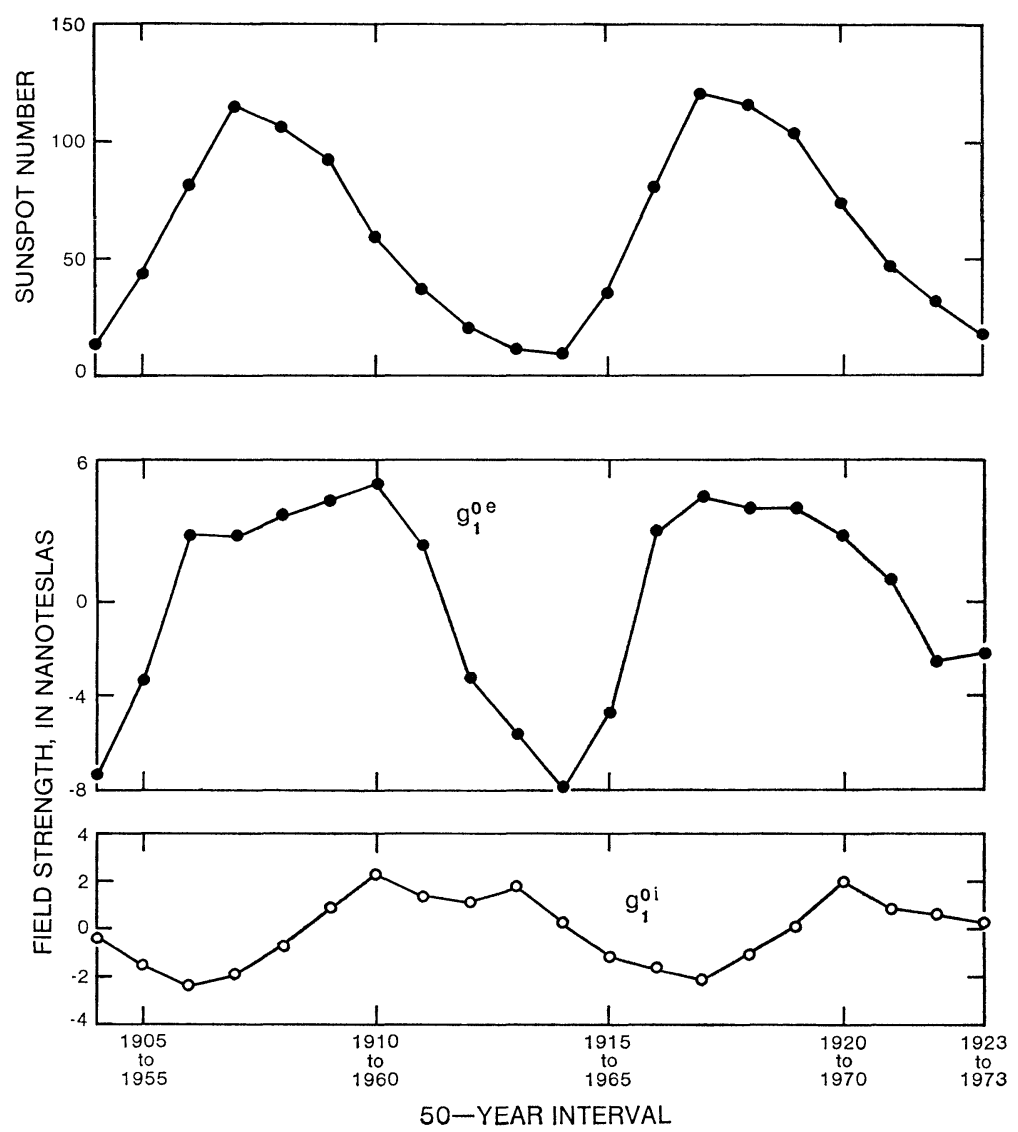

Fig. 9. Variations in the relative sunspot numbers $g_{1}^{0 e}$ and $g_{1}^{0 i}$ averaged at 10-year intervals over 50 years. For example, the points for 1905-1955 are averages of 1905, 1915, 1925, 1935, 1945, and 1955. 


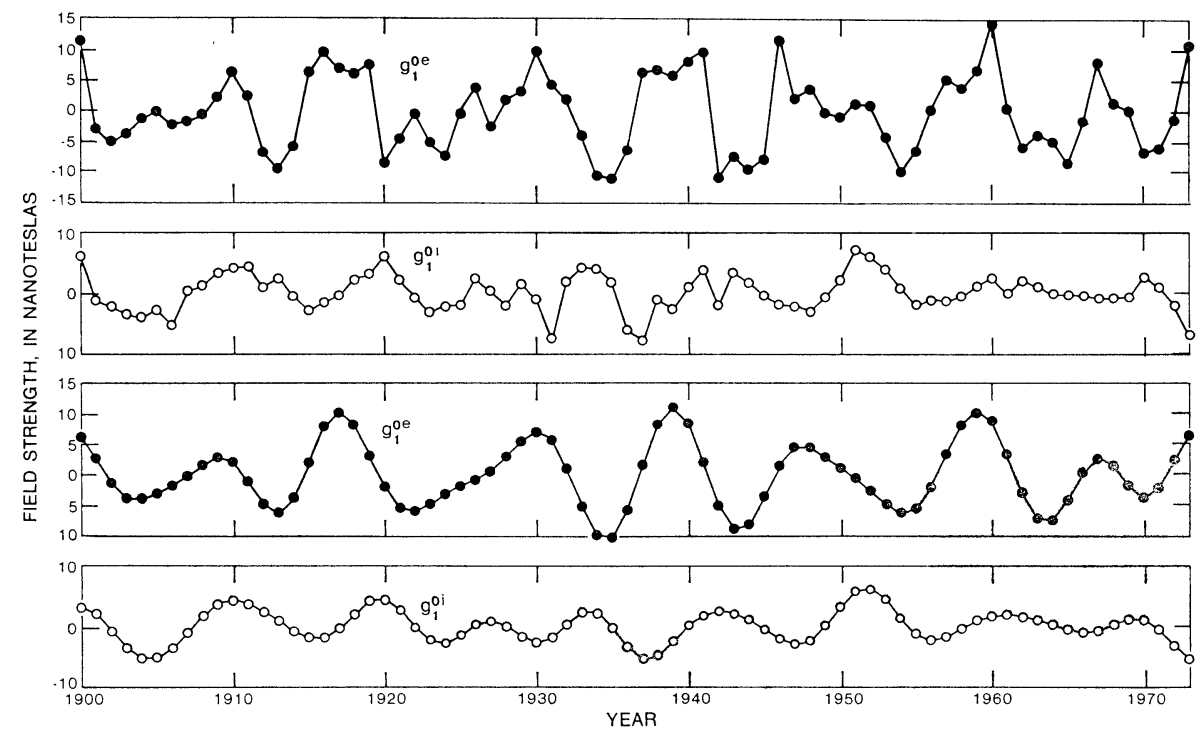

Fig. 10. Variations in $g_{1}^{0 e}$ and $g_{1}^{0 i}$. The top two curves are the variations in $g_{1}^{0 e}$ and $g_{1}^{0 i}$, which includes short period phenomena. The bottom two curves show the same variations after the band pass filter has been applied.

of 10 years, the shape of $g_{1}^{0 i}$ becomes similar to that of $g_{1}^{0 e}$ with a time lag of about 3 years. In order to see the relationship between the variations in $g_{1}^{0 i}$ and $g_{1}^{00}$ they were passed through a band pass filter whose frequency characteristic has its peak around a 10 -year period. The ends of the data interval were treated generally as described for the case of high-pass filtering. The variations before and after the application of the band pass filter are shown in Fig. 10. Highfrequency phenomena have been well smoothed out in the bottom two curves. If the peak-to-peak or trough-to-trough correspondence between the internal and external curves is examined, a good correlation may be noticed with a slight time lag. The variation in $g_{1}^{0 i}$ is, on an average, about 3 years behind $g_{1}^{0 e}$. This is equivalent to the result obtained from Fig. 9. The time lag is so constant during the whole period that, in spite of its small amplitude, the variation in $g_{1}^{0 i}$ may be regarded as a real change which is probably induced by the $g_{1}^{0 e}$ variation.

\section{Fourier Analysis of the Variation in the First-Order Components}

In order to see the quantitative relationship between external and internal parts of the solar cycle variations, a Fourier analysis was made of the data from 1900 to 1973 shown in the two top curves of Fig. 10, which had been passed 
through the high-pass filter with a cut off period around 10 years. The cosine and sine terms were computed respectively by the following equations.

$$
A(\omega)=\frac{2}{73} \sum_{n=0}^{73} g(t) \cos (n \omega \Delta t),
$$

and

$$
B(\omega)=\frac{2}{73} \sum_{n=0}^{73} g(t) \sin (n \omega \Delta t),
$$

where $g(t)$ is either $g_{1}^{0 i}$ or $g_{1}^{0 e}$. The $\omega$ is the angular frequency, and $\Delta t=1$ year in the present case.

The amplitude $C(\omega)$ was computed for $A(\omega)$ and $B(\omega)$, and is shown in Fig. 11. A peak for the 10-year period is remarkable in the external field $g_{1}^{0 e}$. The amplitude amounts to $5.5 \mathrm{nT}$. Relating to the solar cycle variation, a peak around the 5.1-year period is also conspicuous. The internal field variation also has its peak at 10.5 years, but the 5 -year period phenomenon is hardly recognizable.

It should be noted that a significant amount of energy is contained in a 7year period band of the external field variation. However, the cause of this phenomenon is completely unknown.

In order to avoid the effect of finite values at the beginning and the end

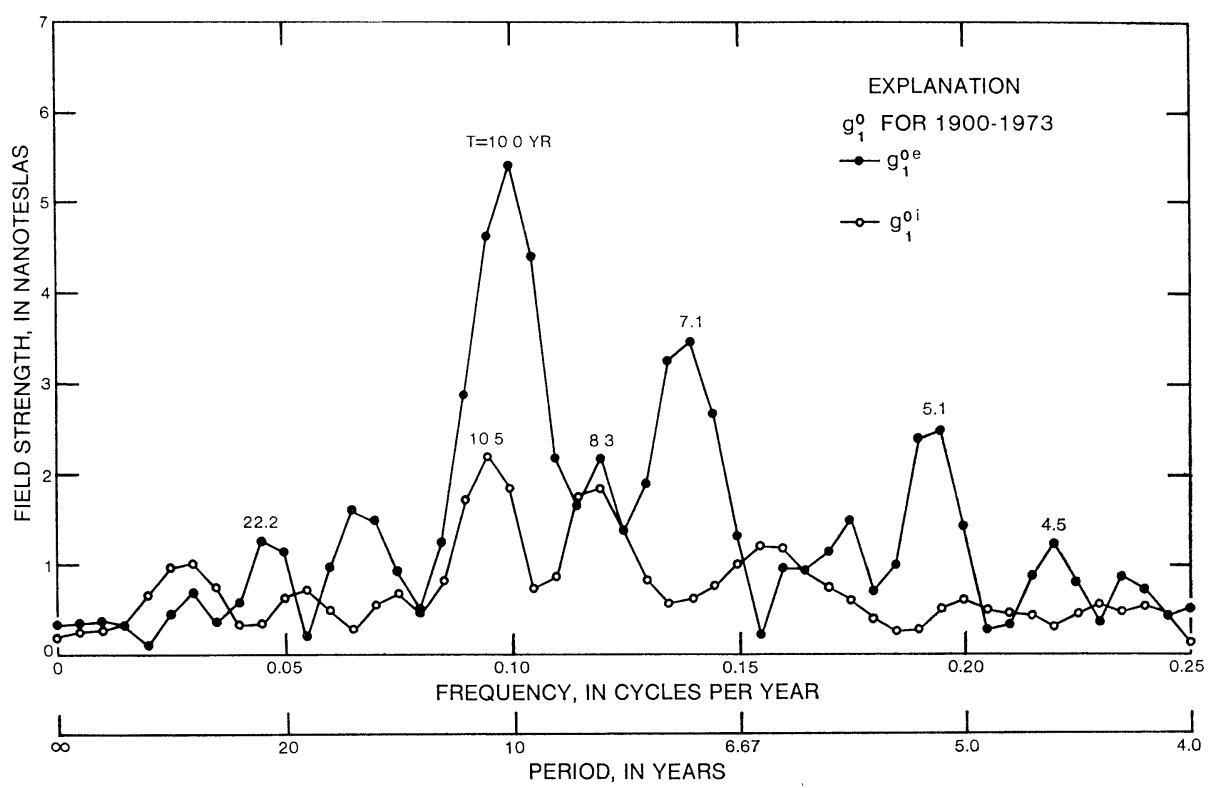

Fig. 11. The results of Fourier analysis for the high passed data. Solid circles are the results for $g_{1}^{0 e}$, and the open circles the results for $g_{1}^{0 i}$. Periods in years are given for the conspicuous peaks. 
Table 6. Ten year components of the external $\left(g_{1}^{0 e}\right)$ and the internal field variation $\left(g_{1}^{0 i}\right)$ obtained by a Fourier transformation.

\begin{tabular}{|c|c|c|c|c|c|c|c|c|c|c|}
\hline \multirow{3}{*}{$\begin{array}{l}\text { Time } \\
\text { (years) }\end{array}$} & \multicolumn{4}{|c|}{ Unweighted } & \multicolumn{4}{|c|}{ Sine-weighted } & \multirow{3}{*}{$i / e$} & \multirow{3}{*}{$c-\varepsilon$} \\
\hline & \multicolumn{2}{|c|}{$g_{1}^{0 e}$} & \multicolumn{2}{|c|}{$g_{1}^{0 i}$} & \multicolumn{2}{|c|}{$g_{1}^{0 e}$} & \multicolumn{2}{|c|}{$g_{1}^{0 i}$} & & \\
\hline & $C$ & Theta & $C$ & Theta & $C$ & Theta & $C$ & Theta & & \\
\hline 10.53 & 4.63 & -118.6 & 2.22 & -24.0 & 3.75 & -117.0 & 1.37 & -16.8 & 0.367 & 100.3 \\
\hline 10.00 & 5.41 & -53.1 & 1.85 & 31.7 & 3.84 & -48.7 & 1.23 & 52.7 & 0.317 & 101.4 \\
\hline 9.52 & 4.43 & 16.6 & 0.73 & 107.0 & 3.21 & 23.6 & 0.84 & 138.1 & 0.263 & 114.5 \\
\hline Mean & & & & & & & & & 0.316 & 105.4 \\
\hline
\end{tabular}

$C$ is the amplitude (nT), and Theta is the phase angle in degrees.

point of the time series, a sine function was adopted as a weighting factor to make both ends zero. Then Fourier analyses were repeated with the weighted data. The amplitudes and the phases of the 10-year period bands are listed in Table 6 along with the results for the unweighted data. The weighting process reduces the original amplitude, but yields more stable results, particularly for the phase angles. Accordingly, for calculating the ratio of the internal to the external field variation, we finally adopted the results obtained from the sineweighted data. The amplitude ratios and phase differences were averaged over the 10 -year band, the mean period being 10.5 years, and the modulus $(i / e)$ was found to be 0.32 , with a phase difference $(\iota-\varepsilon)$ of $105^{\circ}$. The phase difference corresponds exactly to a 3 -year lag of $g_{1}^{0 i}$ behind $g_{1}^{0 e}$, which was pointed out in the previous section.

\section{Discussion and Conclusion}

\subsection{The nature of the geomagnetic variation associated with the solar activity}

One of the interesting features revealed by this investigation is the existence of double peaks during one solar cycle in the time variation of $g_{1}^{0 e}$. One peak nearly coincides with the maximum of sunspot number, and the other appears at the declining stage of the solar activity. Recently, a few papers have appeared reporting the double peaks in the geomagnetic activity. As was discussed in a previous section, MeYers and Allen (1977) provided a frequency distribution of magnetically disturbed days for the period from 1930 to 1975 in terms of $A p$ index that clearly indicated two peaks in a solar cycle. Examining the $K p$ index, OL' (1971) noticed that two maxima existed in the magnetic disturbances. He related the first maximum to the frequency of nonrecurrent magnetic storms and the second, which appeared a few years preceding the sunspot minimum, to recurrent disturbances. Sutorik and Cruickshank (1977) examined the $A p$ 
index for the period from 1868 to 1976 and confirmed that the recurrent geomagnetic activity was seen at the declining stage of the solar activity. From examination of the solar magnetic field S. Matsushita (private communication, 1977) suggests the existence of the double peak phenomenon in statistics of the number of days when the away-from-the-sun sector structure was observed on the earth. Certain features of geomagnetic disturbances are likely to be closely related to solar-wind streams from the coronal holes of the sun and to interplanetary fields (Sheeley et al., 1976; SvalgaARD, 1977). Investigation of the physical processes that produce magnetic disturbance is beyond the scope of the present work. One thing, however, we should note here is that the double peak is not a phenomenon that can only be seen in the statistics of the magnetic disturbances, but that it actually exists in the variation in the axial dipole component.

Annual mean values of the geomagnetic components on which our analyses are based are computed from daily means of each component. Magnetic variations with periods shorter than a year contained in the annual mean values are suspected to contaminate the results. There seem to be two possibilities of contamination. One is the effect of disturbances on the mean values. For the investigation of the long-term variation in the geomagnetic field, one of the greatest concerns is whether shorter period disturbances superposed on it may conceal the long-term trend. The second problem is the effect of diurnal variations. The amplitude of $S q$ changes with solar activity (MAtsushita and Maeda, 1965; Malin et al., 1975). If the midnight values are assumed to represent the undisturbed state of the geomagnetic field, the diurnal variation will lower the daily means from the nighttime level, and the amplitude variation in $S q$ due to solar activity can be a cause of solar cycle variation in the annual mean values.

Regarding the first question, no detailed investigation has been made as yet. One way of solving this question is to compare the variations obtained on the quiet-day data with those on the all-day data. A preliminary check of the annual mean values of the three components at Tucson (YukUtake, 1965) suggested little difference between them. Investigation now under way for several observatory data indicates that, though there exists significant difference, the solar cycle variation in the quiet-day annual mean is still large.

The change in the $S q$ amplitude has been discussed by MALIN et al. (1975), and HARWOOD and MALIN (1977), in which the necessity of correction of the amplitude variation in $S q$ was pointed out. Even if the correction is made, a major part of the solar cycle variation is likely to be preserved in the long period variations of the adjusted values.

Differences between the annual means of the nighttime values and those 
of the all-day hourly values were examined on the data obtained at the Kakioka Magnetic Observatory. It has been revealed that the differences range from 1 to $3 \mathrm{nT}$ around the mean of $1.4 \mathrm{nT}$ with a standard deviation of $0.63 \mathrm{nT}$ for the horizontal component and from 4 to $8 \mathrm{nT}$ around the mean of $5.3 \mathrm{nT}$ with a standard deviation $1.07 \mathrm{nT}$ for the vertical component during the period from January 1960 to December 1976, which covers the largest peak-to-trough variation in the external axial component $g_{1}^{0 e}$. The difference in the behavior of the all-day value and the nighttime value is considered to be contained in the standard deviations rather than in the mean value of the difference itself. The standard deviations estimated above are small compared with the peak-to-trough variation in $g_{1}^{0 e}$ computed on the all-day data, about $50 \mathrm{nT}$, which yields about $40 \mathrm{nT}$ variation of the horizontal component at Kakioka. Therefore, it may be said that the nighttime value is also subjected to the solar cycle variations. Although further investigation is needed on the problem of the contamination of short period phenomena into the annual mean values, most of the solar cycle variations in the annual mean values may be expected to represent the longterm change in the geomagnetic field.

\subsection{The relationship between the external and the internal terms}

The ratio of the internal to the external coefficients provides important information regarding the distribution of the electrical conductivity in the Earth, provided that the internal term is entirely due to the field induced by the external field variations. Phenomena with periods as long as 10 years supply particularly important information about the deeper part of the mantle. The ratios of $g_{1}^{0 i} / g_{1}^{0 e}$ obtained by other analyses for the solar cycle variation of "11 years" are listed in Table 7 together with the results of the present work. Courtillot and LEMouËL (1976) gave only the 11-year harmonics of the $X$ and $Z$ components. The ratio i/e given in the table was calculated from these values. The results seem to be quite consistent with each other. The ratios range from 0.26 to 0.34 , except for those of YukUtake (1965) and HARwood and Malin (1977) who give the low value as 0.16 and 0.12 respectively. The phase angle does not

Table 7. The ratios of the internal to external parts of the "11-year" period variation.

\begin{tabular}{llr}
\hline & Modulus $(i / e)$ & Phase $(i / e)$ \\
\hline YUKUTAKe (1965) & $0.16 \pm 0.08$ & $96^{\circ}$ \\
COURTILLOT and LEMOUËL (1976) & 0.26 & $100^{\circ}$ \\
ALLDREDGE (1976) & $0.34 \pm 0.13$ & $30^{\circ}$ \\
HARWOOD and MALIN (1977) & $0.12 \pm 0.07$ & $8 \pm 30^{\circ}$ \\
ISIKARA (1977) & 0.28 & $77^{\circ}$ \\
The present work & 0.32 & $105^{\circ}$ \\
\hline
\end{tabular}


scatter very much either; it ranges from $77^{\circ}$ to $105^{\circ}$ if AlldREDGE's (1976) and HARWOOD and MALIN's (1977) figures are discarded.

It is necessary to consider again the effect of $S q$. If the effect of $S q$ on the all-day mean is not ignorable, the ratio would be affected. HARWOOD and MALIN (1977) made an adjustment of the all-day means to the midnight values. The adjustment reduced the amplitude of the external and the internal coefficients by about $20 \%$. However, the ratio of the internal to the external by this adjustment was changed only from 0.13 to 0.12 in their case. Since the specific process of their adjustment is not very clear to us, we made no such adjustment.

In the present work, the analyses for the recent data set (1940-1973), which is considered more accurate than the older one, gave variations in the internal coefficient that were too small to permit us to derive a reliable ratio between the internal and external fields. It is not known whether this shortcoming is due to the errors that might be incidently included in the data or to some other cause. In spite of the fair convergence of the various analyses in Table 7, they cannot yet be said to be conclusive. Only the accumulation of accurate data will be able to clarify the ambiguity.

We are grateful to M. Sugiura for providing his numerical filters. We have benefited by discussion with L.R. Alldredge, J.H. Allen, and S. Matsushita. Discussion with K. Svendsen on the observatory data was useful. We would like to express our thanks to C. Kisslinger for his interest and support. This work would not have been possible without the assistance of the National Geophysical and Solar-Terrestrial Data Center of the National Oceanographic and Atmospheric Administration, Boulder, Colorado, and the assistance of the Office of Applications of the National Aeronautics and Space Administration under Grant NAS-W-2129.

\section{REFERENCES}

AlldRedge, L.R., Effects of solar activity on annual means of geomagnetic components, $J$. Geophys. Res., 81, 2990-2996, 1976.

Alldredge, L.R., Geomagnetic variations with periods from 13 to 30 years, J. Geomag. Geoelectr., 29, 123-135, 1977.

Alldredge, L.R., C.O. Stearns, and M. Sugiura, The determination of geomagnetic external spherical harmonic coefficients, J. Geomag. Geoelectr., 31, 495-508, 1979.

Barraclough, D.R., J.M. Harwood, B.R. Leaton, and S.R.C. Malin, A model of the geomagnetic field at epoch 1975, Geophys. J. R. Astr. Soc., 43, 645-659, 1975.

CAIN, J.C., Models of the earth's magnetic field, in Radiation Trapped in the Earth's Magnetic Field, edited by B. McCormac, pp. 570-587, Gordon and Breach, New York, 1966.

CAIN, J.C. and S.J. CAIN, Derivation of the international geomagnetic reference field, NASA TND-6237, 1971, Available from U.S. Nat1. Tech. Inf. Svc., Springfield, Va. 22151, as rep. NASA-TMX-65604, 1971.

CAIN, J.C. and W.M. DAvis, Low latitude variations of the magnetic field, in Symposium on low level satellite surveys, IAGA report from 2nd General Scientific Assembly, Sept., 1973. Abstract also in Intern. Assoc. of Geomag. and Aeron. Bulletin No. 34, pp. 264-265, 1973. 
CAin, J.C., W.E. Daniels, S.J. Hendricks, and D.C. Jensen, An evaluation of the main geomagnetic field, 1940-1962, J. Geophys. Res., 70, 3647-3674, 1965.

CAIn, J.C., S.J. Hendricks, R.A. LANGel, and W.V. Hudson, A proposed model for the international geomagnetic reference field-1965, J. Geomag. Geoelectr., 19, 335-355, 1967.

Chapman, S. and J. Bartels, Geomagnetism, 1049 pp., Clarendon Press, Oxford, 1940.

COURTILLOT, V. and J.L. LEMOUËL, On the long-period variations of the earth's magnetic field from 2 months to 20 years, J. Geophys. Res., 81, 2941-2950, 1976.

CURrie, R.G., Geomagnetic line spectra-2 to 70 years, Astrophys. Space Sci., 21, 425-438, 1973.

Forbush, S.E., The geomagnetic field of the equatorial ring current, in Carnegie Institution of Washington Year Book 63, pp. 351-354, Carnegie Institution of Washington, Washington, 1964.

HARWOOD, J.M. and S.R.C. MALin, Sunspot cycle influence on the geomagnetic field, Geophys. J. R. Astr. Soc., 50, 605-619, 1977.

IsIKARA, A.M., Solar cycle controlled variation of the geomagnetic field, Acta Geodaet. Geophys. Montanist. Acad. Sci. Hung., 12, 397-405, 1977.

Malin, S.R.C., A. Cecere, and A. PAlumbo, The sunspot cycle influence on lunar and solar daily geomagnetic variations, Geophys. J. R. Astr. Soc., 41, 115-126, 1975.

Matsushita, S. and H. MAeda, On the solar quiet day variation field during the IGY, $J$. Geophys. Res., 70, 2559-2578, 1965.

Meyers, H. and J.H. Allen, Some summary geomagnetic activity data; 1932-1976, National Geophysical and Solar-Terrestrial Data Center (Boulder, Co.), Data fact sheet No. 1, pp. 1-12, 1977.

Nie, Norman H., C.H. Hall, J.G. Jenkins, K. Steinbrenner, and D.H. Brent, SPSS (Statistical Package for the Social Sciences), 675 pp., McGraw-Hill, New York, 1975.

OL', A.I.. Physics of the 11 year variation of magnetic disturbances, Geomag. Aeron., 11, 549551 and $647,1971$.

Peddie, N.W. and E.B. Fabiano, A model of the geomagnetic field for 1975, J. Geophys. Res., 81, 2539-2542, 1976.

Regan, R.D., J.C. CAIN, and W.M. DAvis, A global magnetic anomaly map, J. Geophys. Res., 80, 794-802, 1975.

Sheeley, N.R., Jr., J.W. HaRveY, and W.C. Feldman, Coronal holes, solar wind streams, and recurrent geomagnetic disturbances; 1973-1976, Solar Phys., 49, 278, 1976.

Sugiura, M. and D. Poros, Hourly values of equatorial Dst, Magnetic tape or printout available from World Data Center A for Solar Terrestrial Physics (Boulder, Co.), November, 1977.

Sutorik, J.A. and C.M. CRuickshank, A division of the $a a$ indices into six classes basen on the Ap index, 1868-1976, U.S. Natl. Oceanog. Atmos. Admin. Tech. Rpt., ERL 389-SEL-38, 1-11, 1977.

SvalgaARD, L., Geomagnetic activity: Dependence on solar wind parameters, Inst. Plasma Res., Stanford Univ. (SUIPR), No. 699, 1-65, 1977.

YUKUTAKe, T., The solar cycle contribution to the secular change in the geomagnetic field, $J$. Geomag. Geoelectr., 17, 287-309, 1965.

YukUtake, T., Power spectrum analysis of the geomagnetic secular variation, Internat. Assoc. Geomag. and Aeronomy Bull., No. 34, 86, 1973.

Yukutake, T., W.I. Paddock, and J.H. Allen, Annual mean values of geomagnetic components for selected observatories, 1940-73, World Data Center A for Solid Earth Geophysics, Report, SE-17, 1-81, 1979. 